\title{
VOLTAGE REGULATION OF DC-MICROGRID WITH PV AND BATTERY
}

by

JIWEI SUN

Submitted in partial fulfillment of the requirements

For the degree of Master of Science

Department of Electrical Engineering and Computer Science CASE WESTERN RESERVE UNIVERSITY

May, 2017 


\section{Voltage Regulation of DC-Microgrid with PV and Battery \\ Case Western Reserve University \\ Case School of Graduate Studies}

We hereby approve the thesis ${ }^{1}$ of

\section{JIWEI SUN}

for the degree of

Master of Science

\section{Dr. Wei Lin}

Committee Chair, Adviser

Department of Electrical Engineering and Computer Science

\section{Dr. Mingguo Hong}

Committee Member

$03 / 27$

Department of Electrical Engineering and Computer Science

\section{Dr. Kenneth Loparo}

Committee Member

$03 / 27$

Department of Electrical Engineering and Computer Science

\footnotetext{
${ }^{1}$ We certify that written approval has been obtained for any proprietary material contained therein.
} 
Dedicated to my parents with

Love and Gratitude 


\section{Table of Contents}

List of Figures $\quad$ vi

Acknowledgements vii

Abstract $\quad$ viii

Chapter 1. Introduction 1

Chapter 2. System Configuration and

DC-microgrid Model 4

$\begin{array}{ll}\text { Photovoltaic panel model } & 5\end{array}$

$\begin{array}{ll}\text { Battery model } & 6\end{array}$

$\begin{array}{ll}\text { Load model } & 7\end{array}$

Chapter 3. Problem Formulation 99

Control Signals $\quad 9$

$\begin{array}{ll}\text { Dynamic Model } & 10\end{array}$

$\begin{array}{ll}\text { State Space Model } & 10\end{array}$

Equilibrium Point 11

$\begin{array}{ll}\text { Translation } & 12\end{array}$

$\begin{array}{ll}\text { Control Objectives } & 12\end{array}$

$\begin{array}{lll}\text { Chapter 4. Stability Analysis } & 14\end{array}$

$\begin{array}{ll}\text { Stability of Unforced System } & 14\end{array}$

$\begin{array}{ll}\text { Definition of Passivity } & 16\end{array}$

$\begin{array}{ll}\text { Passive System Theory } & 16\end{array}$

$\begin{array}{ll}\text { Controller Design } & 18\end{array}$ 
Chapter 5. Output Regulation and Controller Design

Traditional Method for Tracking Problem 22

Nonlinear Regulator Theory and Controller Design 26

Passivity-based Design $\quad 36$

$\begin{array}{lll}\text { Chapter 6. } & \text { Case Studies } & 39\end{array}$

$\begin{array}{ll}\text { Illumination Change } & 39\end{array}$

$\begin{array}{ll}\text { Load Change } & 42\end{array}$

$\begin{array}{lll}\text { Chapter 7. } & \text { Future Study } & 46\end{array}$

System with Disturbance $\quad 46$

$\begin{array}{ll}\text { More Complexed System } & 47\end{array}$

$\begin{array}{ll}\text { More Advanced Control } & 48\end{array}$

$\begin{array}{lll}\text { Chapter 8. } & \text { Conclusion } & 50\end{array}$

$\begin{array}{ll}\text { Complete References } & 52\end{array}$ 


\section{List of Figures}

2.1 The DC-microgrid system configuration 4

2.2 The circuit of DC-microgrid system 5

$\begin{array}{lll}2.3 & \text { I-V curve } & 6\end{array}$

$\begin{array}{lll}2.4 & \text { P-V curve } & 7\end{array}$

6.1 simulation result of illumination changes using constant feedback

$\begin{array}{ll}\text { control } & 40\end{array}$

6.2 simulation result of illumination changes using state feedback control 41

6.3 simulation result of illumination changes using measurement feedback

$\begin{array}{ll}\text { control } & 42\end{array}$

6.4 simulation result of load changes using constant feedback control 43

6.5 simulation result of load changes using state feedback control $\quad 44$

6.6 simulation result of load changes using measurement feedback control 44

7.1 The DC-microgrid system with two PVs and batteries 48 


\section{Acknowledgements}

I would like to thank my supervisor, Dr. Wei Lin, for his support and review of this thesis. His wide knowledge and guidance helps me a lot for this thesis.

My appreciation as well to Professor Mingguo Hong and Professor Kenneth Loparo for their help in discussion which gives me inspiration for this research.

Special thanks to Huiying Zhao, who helps me understand the system configuration, and Matthew Ma who helps me with the grammar check. 


\title{
Abstract \\ Voltage Regulation of DC-Microgrid with PV and Battery
}

\author{
Abstract \\ by \\ JIWEI SUN
}

This MS thesis develops voltage regulation and maximum power point tracking (MPPT) control schemes for a class of DC-microgrids. The DC-microgrid under consideration consists of photovoltaic (PV) panel, battery, constant resistance loads and constant power loads. In this study, a dynamic model of the dc-microgrid system is derived and described by a multi-input and multi-output nonlinear system with nonaffine inputs. Based on the nonlinear dynamic model thus built, we employ the output regulation theory to design a local state feedback control law that regulates voltages to prescribe set points and maximizes the power output from PV. Global set point regulation is also studied by virtue of passive system theory for non-affine systems and the Lyapunov stability theory. Three control strategies, namely, constant feedback, state feedback and measurement feedback, are proposed. The effectiveness of the proposed control schemes is validated by simulations when both illumination change and load change occur. 


\section{Introduction}

A microgrid is an integration of local generators and local loads. When the sources and loads work in DC, it becomes a DC-microgrid. DC-microgrid has advantages over an AC-microgrid in the following aspects: 1)Higher power system efficiency because most systems are are naturally DC, so that fewer DC/AC or AC/DC converter will be used. 2)Higher reliability because fewer converters require fewer electronic components, and with fewer potential of failure. 3)Lower capital cost because of fewer power electronic components are used. 4)Lower control system complexity and higher survivability when subject to disturbances. This is due to the elimination of synchronization requirements of AC systems. The overall introduction can be seen in (Backhaus, 2015).

A DC-microgrid is able to work in either grid connected mode or island mode. When working in grid connected mode, the microgrid can either absorb power from the grid, or supply power to the grid depending on the amount of power it consumes and produces. If the microgrid generates more power than it needs, it can supply to the grid. If the microgrid demands more power than it produces, it can absorb power from the grid. In island mode, the local energy sources need to supply the local loads. 
An example of a DC-microgrid is the International Space Station. The International Space Station has several solar arrays and batteries to supply power while operation system and other devices consume power. When the solar arrays have access to the light, it will provide power for the load and charging the batteries. If the solar arrays cannot generate enough power for the loads, the batteries will discharge to supply energy. Lots of DC-DC converters are used to regulate the voltage and protect the system. The details can be found in (Gietl, 2000).

In this paper, the focus will be DC-microgrid with PV and battery working in island mode. In this case, not only does the load voltage need to be regulated, but also the battery needs to be charged from PV. In order to improve the efficiency of PV, the maximum power point tracking (MPPT) method is required, which is introduced in (Freeman, 2010). The DC-microgrid system becomes a nonlinear system due to the nature of some of the DC sources, DC converters and constant power loads, which makes the system tough to control. For example, the PV output is not a constant source, and has nonlinear property. The stability analysis of PV is discussed in (Tan, Jenkins, 2004), and the stability when PV connects to the grid is researched in (Wang, Lin, 2000) and (Rodriguez, Amaratunga, 2004). Constant power loads are another big problem because they can be viewed as negative impedance and tend to destabilize the converters. The effect of constant power load is discussed in (Kwasinski, Krein, 2007) and (Kwasinski, 2011). For the system without constant power load, some research has been done to solve the voltage regulation problem. (Zhang, 2015) and (Wu, 2016) applies MPPT algorithm to maximum PV output and PI controller to do the voltage tracking; (Becherif, 2007) tries the passivity-based control. The DC-microgrid with constant power load has also been studied. In (Radwan, Mohamed, 2012), the author uses the damping method 
to regulate voltage; compensation transfer function method is used in (Ahmadi, Ferdowsi, 2014); (Gautam, 2015), and (Agarwal, 2015) derives sliding mode control law.

In this study, output regulator theory, which is discussed in (Byrnes, 1997), is used to design the controller for DC-DC converters. A new control law is derived to regulate the DC bus and load voltage under various operating conditions. The load is considered to be a combination of a kind of constant power load and conventional resistive load. To enhance the efficiency, the power output from PV is always required to be maximized. The performance of the proposed controller is verified through MATLAB simulation results.

The outline of the paper as follows: Chapter 2 discusses the system configuration and build the DC-microgrid model; Chapter 3 describes the problem formulation and the control objectives; Chapter 4 provides the stability analysis using Lyapunov stability theorem and passivity-based design; Chapter 5 reviews the traditional control method and output regulation theory and three different control laws are derived; Chapter 6 presents the case studies; Chapter 7 gives possible future directions; and the conclusions are given in Chapter 8. 


\section{System Configuration and DC-microgrid Model}

The configuration of DC-microgrid system is shown in Fig. 2.1. The DC-microgrid consists of a PV panel, a battery storage, a DC bus, several DC/DC converters and two loads that work on different voltage level. A DC/DC boost converter is used between PV and DC bus to maximize the power output from the PV. Battery connects to the DC bus through a bidirectional DC/DC buck-boost converter to regulate the voltage on the DC bus. A bus load directly connects to the DC bus. The other load links to the DC bus through a DC/DC buck converter and can be viewed as a constant power load.

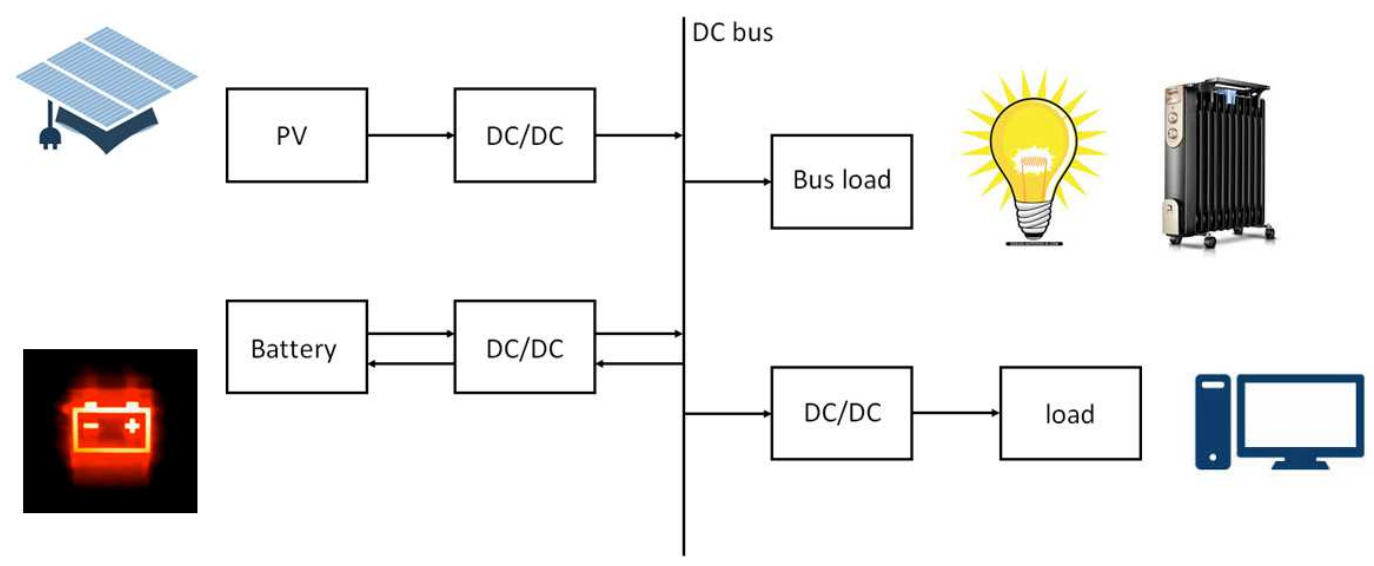

Figure 2.1. The DC-microgrid system configuration

The circuit is shown in figure 2.2. 


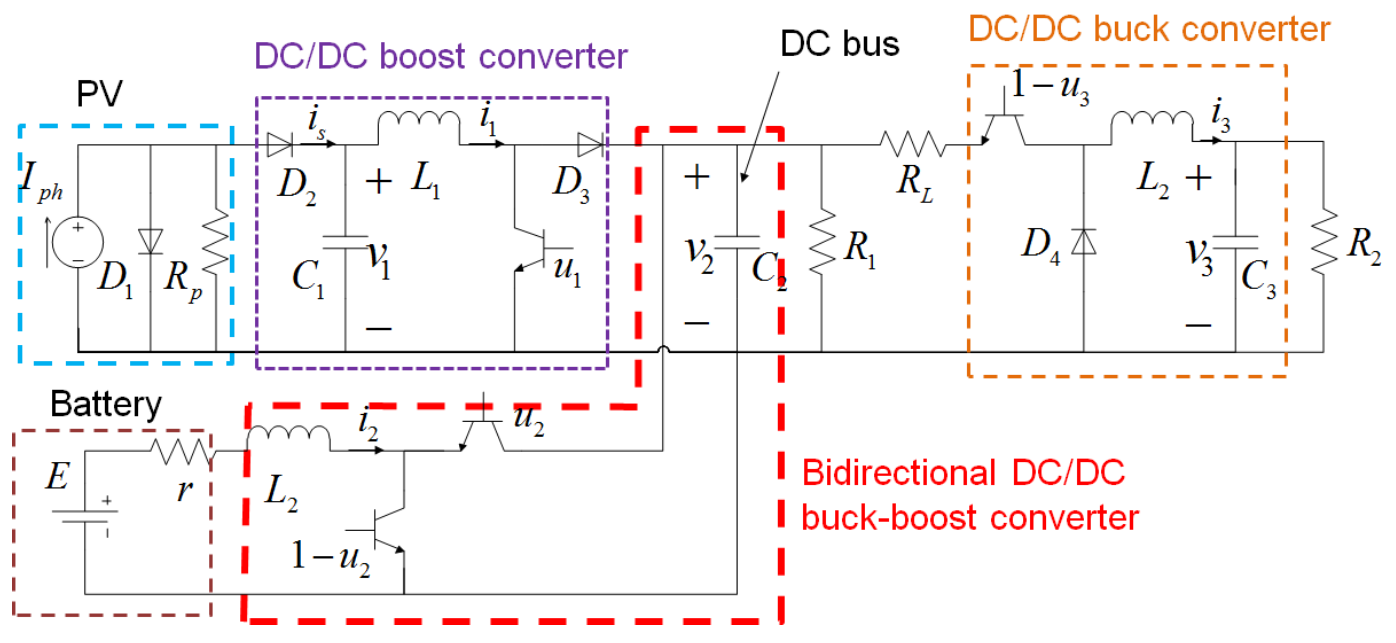

Figure 2.2. The circuit of DC-microgrid system

\subsection{Photovoltaic panel model}

The PV panel is modeled by a current source $I_{p h}$ (which relates to the irradiation and temperature) connected in parallel with a diode and with a shunt resistor $R_{p}$. The resistor represents the disturbance current and $i_{s}$ is the current output of PV panel.

The power output of PV is: $P=i_{s} v_{1}$.

$$
\begin{aligned}
i_{s} & =I_{p h}-i_{D_{1}}-i_{R_{p}} \\
i_{D_{1}} & =I_{0}\left(e^{a v_{1}}-1\right) \\
a & =\frac{q}{n k T}
\end{aligned}
$$

where $I_{0}$ is the reverse saturation of the diode, $q$ is the elementary charge, $T$ is the cell temperature, $k$ is Boltzmann's constant, $n$ is the ideality factor. The I-V and P-V characteristics are shown in Figure 2.3 and Figure 2.4. 


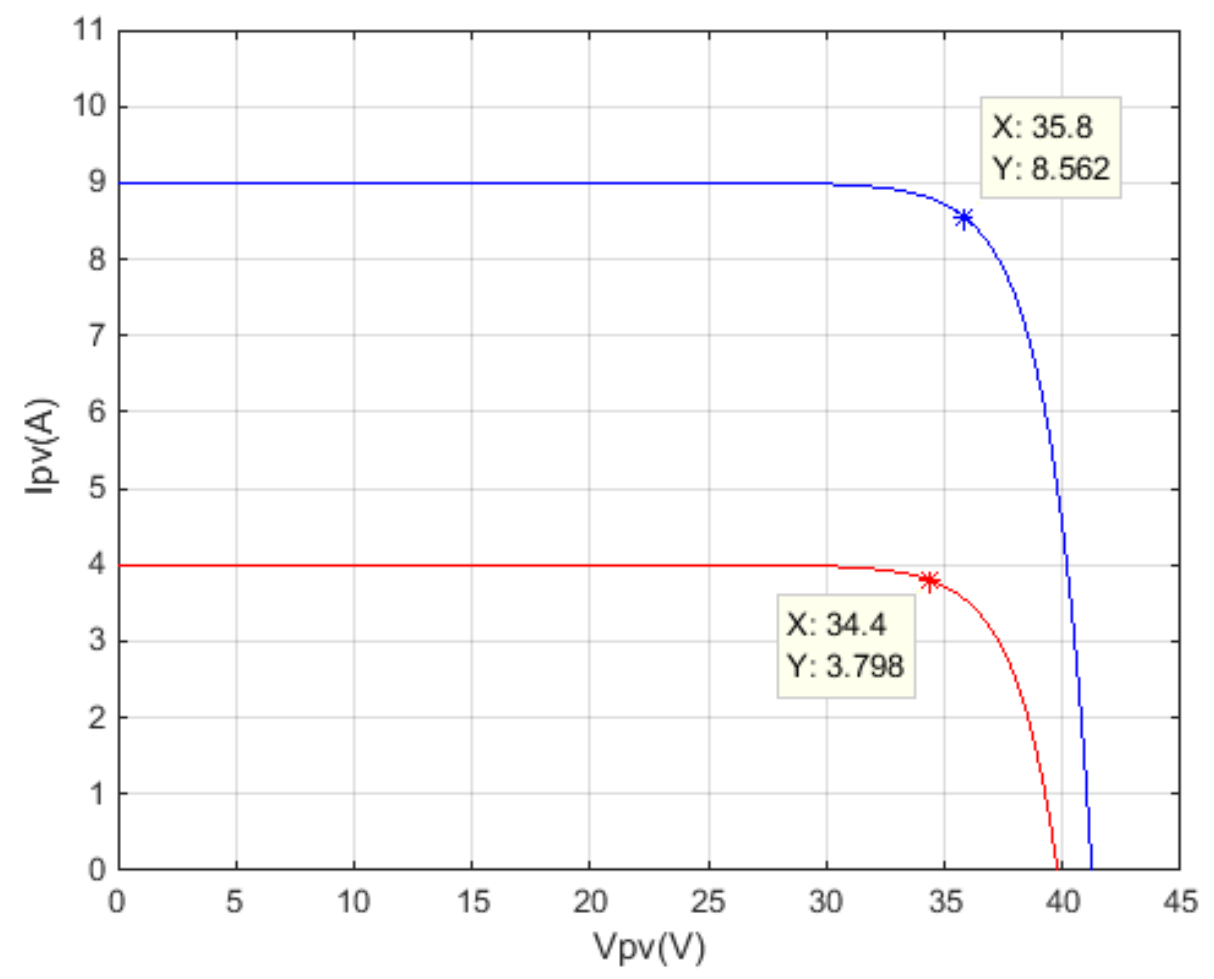

Figure 2.3. I-V curve

In this model, the irradiation and temperature is considered to be constant. Diodes are ideal diodes except $D_{1}$.

A DC/DC boost converter is used to connect the PV panel to the DC bus. The main purpose of this converter is to control the power output of the PV panel.

\subsection{Battery model}

The battery is modeled with an electromotive force $E$ and a series resistor $r$. The battery connects to the DC bus through a DC/DC bidirectional buck-boost converter. This converter serves to regulate the voltage on the DC bus as well as control the charge/discharge of the battery. When the power output of the PV is bigger than the consumption of the 


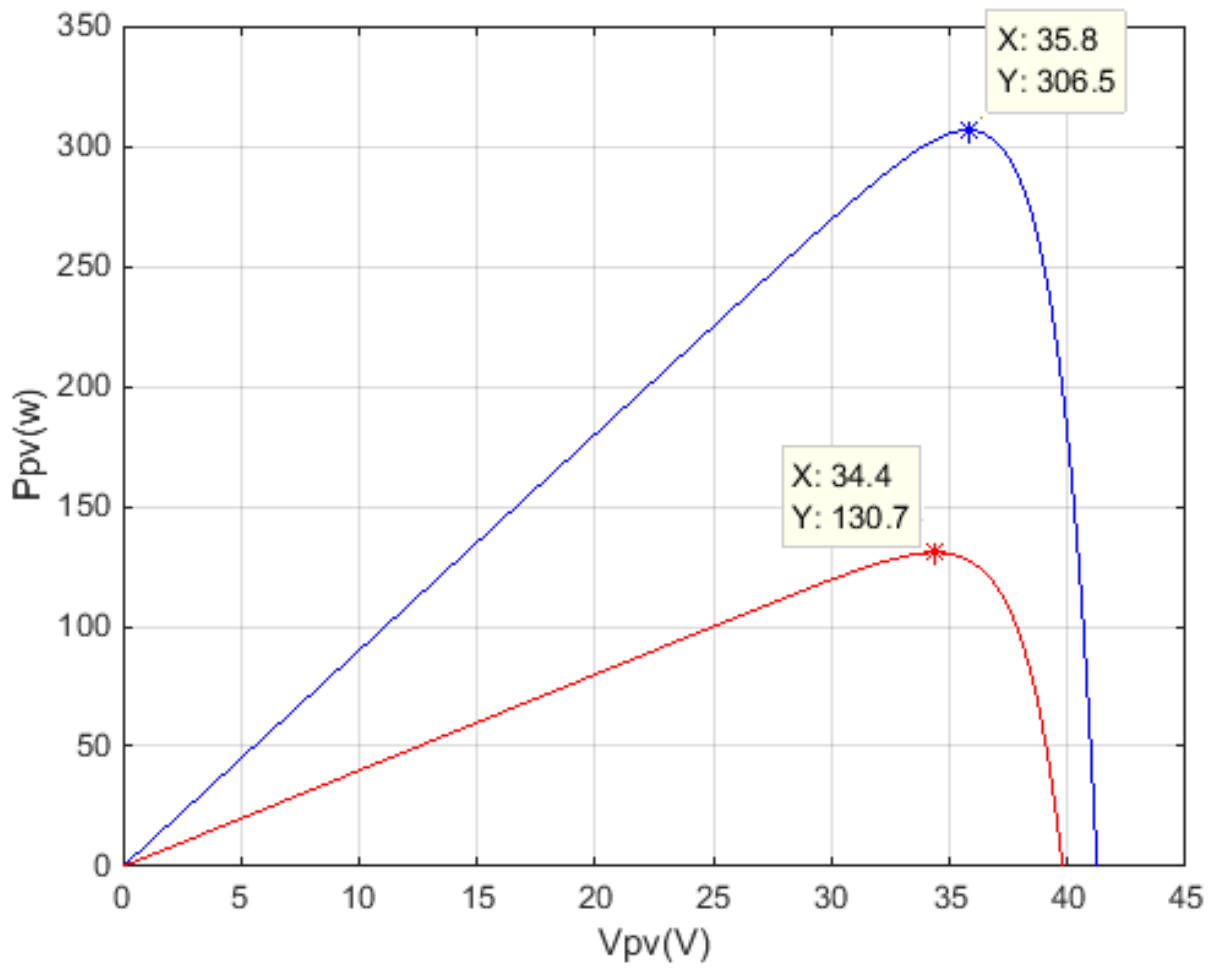

Figure 2.4. P-V curve

load, the battery will absorb the extra power. If the solar power is not enough to serve the loads, the battery will release power to maintain the DC bus voltage.

\subsection{Load model}

There are two loads in the model. $R_{1}$ represents the load that connects to the DC bus directly. The other load $R_{2}$ works on a different voltage level. Thus, a DC/DC buck converter is used for the change of voltage level and regulate the load voltage. Both loads are modeled as constant resister. There is a line resister $R_{L}$ which represents the loss of the transmission line. Since $P=V^{2} / R$, the power consumption of $R_{2}$ is constant. Because 
there's no power loss at the buck converter, the load $R_{2}$ together with the buck converter can be viewed as a constant power load connects to the DC bus. 


\section{Problem Formulation}

\subsection{Control Signals}

There are three controls in the model. The control signals are duty cycles of the converters shown in firgure 2 and have the following restrictions: $0 \leqslant u_{i} \leqslant 1, i=1,2,3$.

The input signal of the transistor is zero $\longrightarrow$ the switch is closed. The input signal of the transistor is one $\longrightarrow$ the switch is open. If the input signal is $1-u$, then $1-u=0 \longrightarrow$ the switch is closed.

$u_{1}$ controls the power output of the PV; $u_{2}$ controls the DC bus voltage and the charge/discharge of the battery; $u_{3}$ controls the load voltage. 


\subsection{Dynamic Model}

Using Kirchhoff's laws, the dynamic model of the system can be written as

$$
\begin{aligned}
L_{1} \frac{d i_{1}}{d t} & =v_{1}-v_{2}\left(1-u_{1}\right) \\
C_{1} \frac{d v_{1}}{d t} & =i_{s}-i_{1}=I_{p h}-I_{0}\left(e^{a v_{1}}-1\right)-\frac{v_{1}}{R_{p}}-i_{1} \\
L_{2} \frac{d i_{2}}{d t} & =E-i_{2} r-v_{2} u_{2} \\
C_{2} \frac{d v_{2}}{d t} & =i_{1}\left(1-u_{1}\right)+i_{2} u_{2}-\frac{v_{2}}{R_{1}}-i_{3}\left(1-u_{3}\right) \\
L_{3} \frac{d i_{3}}{d t} & =\left(v_{2}-i_{3}\left(1-u_{3}\right) R_{L}\right)\left(1-u_{3}\right)-v_{3} \\
C_{3} \frac{d v_{3}}{d t} & =i_{3}-\frac{v_{3}}{R_{2}}
\end{aligned}
$$

with the constraints: $v_{2}>0, v_{3}>0, i_{1} \geqslant 0, i_{3}>0$.

\subsection{State Space Model}

Let $x^{T}=\left[x_{1}, x_{2}, x_{3}, x_{4}, x_{5}, x_{6}\right]=\left[i_{1}, v_{1}, i_{2}, v_{2}, i_{3}, v_{3}\right]$ and the system can be rewritten as state space model

$$
\begin{aligned}
& \dot{x}_{1}=\frac{1}{L_{1}}\left[x_{2}-x_{4}\left(1-u_{1}\right)\right] \\
& \dot{x}_{2}=\frac{1}{C_{1}}\left[I_{p h}-I_{0}\left(e^{a x_{2}}-1\right)-\frac{x_{2}}{R_{p}}-x_{1}\right] \\
& \dot{x}_{3}=\frac{1}{L_{2}}\left[E-r x_{3}-x_{4} u_{2}\right] \\
& \dot{x}_{4}=\frac{1}{C_{2}}\left[-\frac{x_{4}}{R_{1}}+x_{1}\left(1-u_{1}\right)+x_{3} u_{2}-x_{5}\left(1-u_{3}\right)\right] \\
& \dot{x}_{5}=\frac{1}{L_{3}}\left[-x_{6}+x_{4}\left(1-u_{3}\right)-R_{L} x_{5}\left(1-u_{3}\right)^{2}\right] \\
& \dot{x}_{6}=\frac{1}{C_{3}}\left[x_{5}-\frac{x_{6}}{R_{2}}\right]
\end{aligned}
$$


with the constraints: $x_{1} \geqslant 0, x_{4}>0, x_{5}>0, x_{6}>0$.

\subsection{Equilibrium Point}

Let $u^{T}=\left[u_{1}, u_{2}, u_{3}\right]=[0,0,0]$, solve the equations $\dot{x}=0$ to get $x^{\star}$.

$$
\begin{aligned}
x_{2}^{\star}-x_{4}^{\star} & =0 \\
I_{p h}-I_{0}\left(e^{a x_{2}^{\star}}-1\right)-\frac{x_{2}^{\star}}{R_{p}}-x_{1}^{\star} & =0 \\
E-r x_{3}^{\star} & =0 \\
-\frac{x_{4}^{\star}}{R_{1}}+x_{1}^{\star}-x_{5}^{\star} & =0 \\
-x_{6}^{\star}+x_{4}^{\star}-R_{L} x_{5}^{\star} & =0 \\
x_{5}^{\star}-\frac{x_{6}^{\star}}{R_{2}} & =0
\end{aligned}
$$

$$
\begin{array}{r}
x_{1}^{\star}=\frac{R_{1}+R_{2}+R_{L}}{R_{1} R_{2}} x_{6}^{\star} \\
x_{2}^{\star}=x_{4}^{\star}=\frac{R_{2}+R_{L}}{R_{2}} x_{6}^{\star} \\
x_{3}^{\star}=\frac{E}{r} \\
x_{5}^{\star}=\frac{x_{6}^{\star}}{R_{2}} \\
I_{p h}-I_{0}\left(e^{\left.a x_{2}^{\star}-1\right)-\frac{x_{2}^{\star}}{R_{p}}-x_{1}^{\star}=0}\right. \\
x^{\star T}=\left[x_{1}^{\star}, x_{2}^{\star}, x_{3}^{\star}, x_{4}^{\star}, x_{5}^{\star}, x_{6}^{\star}\right]^{T}
\end{array}
$$




\subsection{Translation}

Translation means to move the equilibrium points of the system to the origin. Let $\bar{x}=$ $x-x^{\star}$ and rewrite the state equations.

$$
f(\bar{x}, u)=\left[\begin{array}{c}
\frac{1}{L_{1}}\left(\bar{x}_{2}-\bar{x}_{4}+\left(\bar{x}_{4}+x_{4}^{\star}\right) u_{1}\right) \\
\frac{1}{C_{1}}\left(-\bar{x}_{1}-\frac{\bar{x}_{2}}{R_{p}}-I_{0} e^{a x_{2}^{\star}}\left(e^{a \bar{x}_{2}}-1\right)\right) \\
\frac{1}{L_{2}}\left(-r \bar{x}_{3}-\left(\bar{x}_{4}+x_{4}^{\star}\right) u_{2}\right) \\
\frac{1}{C_{2}}\left(\bar{x}_{1}-\frac{\bar{x}_{4}}{R_{1}}-\bar{x}_{5}-\left(\bar{x}_{1}+x_{1}^{\star}\right) u_{1}\right. \\
\left.+\left(\bar{x}_{3}+x_{3}^{\star}\right) u_{2}+\left(\bar{x}_{5}+x_{5}^{\star}\right) u_{3}\right) \\
\frac{1}{L_{3}}\left(\bar{x}_{4}-R_{L} \bar{x}_{5}-\bar{x}_{6}+\left(2 R_{L}\left(\bar{x}_{5}+x_{5}^{\star}\right)\right.\right. \\
\left.\left.-\left(\bar{x}_{4}+x_{4}^{\star}\right)\right) u_{3}-R_{L}\left(\bar{x}_{5}+x_{5}^{\star}\right) u_{3}^{2}\right) \\
\frac{1}{C_{3}}\left(\bar{x}_{5}-\frac{\bar{x}_{6}}{R_{2}}\right)
\end{array}\right]
$$

with the constraints: $\bar{x}_{1} \geqslant-x_{1}^{\star}, \bar{x}_{4}>-x_{4}^{\star}, \bar{x}_{5}>-x_{5}^{\star}, \bar{x}_{6}>-x_{6}^{\star}$.

\subsection{Control Objectives}

The control objectives are to maximum the power output of PV panel, regulate the DC bus voltage and load voltage. The DC bus voltage and load voltage are scheduled constants. However, the maximum power output needs to be decided. Based on the model formulation, the power output

$$
P=\left(I_{p h}-I_{0}\left(e^{a \nu_{1}}-1\right)-\frac{v_{1}}{R_{p}}\right) \nu_{1}
$$

Since $P$ only depends on $v_{1}$ and $P$ is continuous, one can find maximum $P$ by calculating

$$
\frac{\partial P}{\partial v_{1}}=\left(-a I_{0} e^{a v_{1}}-\frac{1}{R_{p}}\right) \nu_{1}+I_{p h}-I_{0}\left(e^{a v_{1}}-1\right)-\frac{v_{1}}{R_{p}}=0
$$




$$
a I_{0} v_{1} e^{a v_{1}}+I_{0} e^{a v_{1}}+\frac{2}{R_{p}} v_{1}=I_{p h}-I_{0}
$$

By solving the equation, the solution $v_{1}=v_{1}^{\star}$ can achieve the maximum power output.

Therefore, the control objectives are $v_{1} \longrightarrow v_{1}^{\star}, v_{2} \longrightarrow v_{2}^{\star}, v_{3} \longrightarrow v_{3}^{\star}$. 


\section{Stability Analysis}

In this chapter, the stability analysis of the system is discussed. Lyapunov stability theory can be used to decide the stability of unforced system. Because the system has certain property, it's a good example to apply the passivity-based design. The definition of passivity and passive system theory is introduced and the control law is designed.

\subsection{Stability of Unforced System}

The system without control $(u=0)$ is globally asymptotically stable at $x=0$. When $u=0$, the system becomes

$$
f(\bar{x}, 0)=\left[\begin{array}{l}
\frac{1}{L_{1}}\left(\bar{x}_{2}-\bar{x}_{4}\right) \\
\frac{1}{C_{1}}\left(-\bar{x}_{1}-\frac{\bar{x}_{2}}{R_{p}}-I_{0} e^{a x_{2}^{\star}}\left(e^{a \bar{x}_{2}}-1\right)\right) \\
\frac{1}{L_{2}}\left(-r \bar{x}_{3}-\bar{x}_{4}\right) \\
\frac{1}{C_{2}}\left(\bar{x}_{1}-\frac{\bar{x}_{4}}{R_{1}}-\bar{x}_{5}\right) \\
\frac{1}{L_{3}}\left(\bar{x}_{4}-R_{L} \bar{x}_{5}-\bar{x}_{6}\right) \\
\frac{1}{C_{3}}\left(\bar{x}_{5}-\frac{\bar{x}_{6}}{R_{2}}\right)
\end{array}\right]
$$

It can be shown with the Lyapunov function

$$
V(\bar{x})=\frac{1}{2} L_{1} \bar{x}_{1}^{2}+\frac{1}{2} C_{1} \bar{x}_{2}^{2}+\frac{1}{2} L_{2} \bar{x}_{3}^{2}+\frac{1}{2} C_{2} \bar{x}_{4}^{2}+\frac{1}{2} L_{3} \bar{x}_{5}^{2}+\frac{1}{2} C_{3} \bar{x}_{6}^{2}
$$




$$
\begin{aligned}
\dot{V}(\bar{x})= & L_{1} \bar{x}_{1} \dot{\bar{x}}_{1}+C_{1} \bar{x}_{2} \dot{\bar{x}}_{2}+L_{2} \bar{x}_{3} \dot{\bar{x}}_{3}+C_{2} \bar{x}_{4} \dot{\bar{x}}_{4}+L_{3} \bar{x}_{5} \dot{\bar{x}}_{5}+C_{3} \bar{x}_{6} \dot{\bar{x}}_{6} \\
= & \bar{x}_{1} \bar{x}_{2}-\bar{x}_{1} \bar{x}_{4}-\bar{x}_{1} \bar{x}_{2}-\frac{1}{R_{p}} \bar{x}_{2}^{2}-I_{0} e^{a x_{2}^{\star}}\left(e^{a \bar{x}_{2}}-1\right) \bar{x}_{2}-r \bar{x}_{3}^{2} \\
& -\bar{x}_{3} \bar{x}_{4}+\bar{x}_{1} \bar{x}_{4}-\frac{1}{R_{1}} \bar{x}_{4}^{2}-\bar{x}_{4} \bar{x}_{5}+\bar{x}_{4} \bar{x}_{5}-R_{L} \bar{x}_{5}^{2} \\
& -\bar{x}_{5} \bar{x}_{6}+\bar{x}_{5} \bar{x}_{6}-\frac{1}{R_{2}} \bar{x}_{6}^{2} \\
= & -\frac{1}{R_{p}} \bar{x}_{2}^{2}-I_{0} e^{a x_{2}^{\star}}\left(e^{a \bar{x}_{2}}-1\right) \bar{x}_{2}-r \bar{x}_{3}^{2}-\frac{1}{R_{1}} \bar{x}_{4}^{2}-R_{L} \bar{x}_{5}^{2}-\frac{1}{R_{2}} \bar{x}_{6}^{2}
\end{aligned}
$$

Since $\dot{V}$ is nonpositive, system without control is stable in the sense of Lyapunov. By La Salle's Invariance Principle,

$$
\dot{V}(\bar{x})=0 \rightarrow \bar{x}_{2}=\bar{x}_{3}=\bar{x}_{4}=\bar{x}_{5}=\bar{x}_{6}=0 \rightarrow \dot{\bar{x}}_{2}=0 \rightarrow \bar{x}_{1}=0
$$

Therefore, system without control is globally asymptotically stable.

The system is a good example for passivity-based stability analysis. Consider the case when $r=0$. This could be using an ideal battery model or the value of $r$ is small enough comparing to the loads. In this case, the unforced system is no longer asymptotically stable. The only conclusion we can get is that the system is stable in the sense of Lyapunov. The passivity-based design is powerful to solve the problem with such property.

In fact, many circuit systems and power systems are passive systems and can use the following method to design the controller. The advantage of passivity-based design is that it can deal with certain nonlinear system which has multi-input/multi-output and is nonaffine. The control signal can be very small and the control law can make the system globally asymptotically stable. 


\subsection{Definition of Passivity}

Consider the nonlinear system

$$
\begin{aligned}
\dot{x} & =f(x, u) \\
y & =h(x, u)
\end{aligned}
$$

An input-output system $\Sigma$ of the form (4.2.5) (4.2.6) is said to be passive if there exists a $C^{0}$ (continuous) nonnegative functions $V: \mathbb{R}^{n} \rightarrow \mathbb{R}$, with $V(0)=0$, such that

$$
V(x(t))-V\left(x_{0}\right) \leqslant \int_{0}^{t} y^{T}(s) u(x) d s \quad \forall u \in \mathbb{R}^{m}, \quad \forall x_{0} \in \mathbb{R}^{n},
$$

where $x(t)$ is a solution of $\dot{x}=f(x, u)$ starting from $x(0)=x_{0}$.

If $V$ is a $C^{r} \quad r \geqslant 1$ function, the passivity inequality is equivalent to

$$
\dot{V} \leqslant y^{T} u \quad \forall u \in \mathbb{R}^{m}
$$

\subsection{Passive System Theory}

The details and proof of this section can be seen in (Lin, 1996). In this paper, the symbol $L_{f} V$ represents Lie derivative, which equals to $\frac{\partial V}{\partial x} f(x)$.

A smooth nonlinear system $\dot{x}=f(x, u)$ can always be represented as

$$
\dot{x}=f_{0}(x)+g_{0}(x) u+R(x, u) u=f_{0}(x)+g_{0}(x) u+\sum_{i=1}^{m} u_{i}\left(R_{i}(x, u) u\right)
$$


where

$$
\begin{aligned}
& f_{0}(x)=f(x, 0) \\
& g_{i}^{0}(x)=g_{i}(x, 0)=\frac{\partial f}{\partial u_{i}}(x, 0) \\
& g_{0}(x)=\frac{\partial f}{\partial u}(x, 0)=\left[\begin{array}{lll}
g_{1}^{0}(x) & \ldots & g_{m}^{0}(x)
\end{array}\right] \in \mathbb{R}^{n \times m}
\end{aligned}
$$

H4.1:there exists a $C^{r} \quad r \geqslant 1$ function $V: \mathbb{R}^{n} \rightarrow \mathbb{R}$, with $V(0)=0$, which is positive definite and proper on $\mathbb{R}^{n}$, such that the unforced dynamic system $\dot{x}=f(x, 0) \triangleq f_{0}(x)$ is Lyapunov stable, i.e. $L_{f_{0}} V(x) \leqslant 0 \quad \forall x_{0} \in \mathbb{R}^{n}$

H4.2:system is zero-state detectable.

A system is called zero-state detectable if

$$
u(t)=0, \quad y=0, \quad \forall t \geqslant 0 \quad \Rightarrow \lim _{t \rightarrow \infty} x(t)=0
$$

Theorem: consider a multi-input non-affine nonlinear system of the form

$$
\begin{array}{r}
\dot{x}=f_{0}(x)+\sum_{i=1}^{m} g_{i}^{0}(x) u_{i}+\sum_{i_{1}=1}^{m} \sum_{i_{2}=1}^{m} g_{i_{1} i_{2}}(x) u_{i_{1}} u_{i_{2}} \\
+\ldots+\sum_{i_{1}=1}^{m} \ldots \sum_{i_{l}=1}^{m} g_{i_{1} \ldots i_{l}}(x) u_{i_{1}} \ldots u_{i_{l}}
\end{array}
$$

Suppose that H4.1 and H4.2 holds. Then the system (4.3.10) is GAS by arbitrarily small smooth state feedback. In particular, a possible choice is

$$
u(x)=-\alpha(x) \frac{\left[L_{g_{0}} V(x)\right]^{T}}{1+\left\|L_{g_{0}} V(x)\right\|^{2}}
$$

where $\alpha(x)$ can either be the function

$$
\alpha(x)=\frac{\frac{\beta}{m}}{1+\left\|\frac{\partial V}{\partial x}\right\|^{2} \rho^{2}(x)} \text { for any } 0<\beta<1,
$$

with

$$
\rho(x) \geqslant \sum_{k=2}^{l}\left[\sum_{i_{1}=1}^{m} \ldots \sum_{i_{k}=1}^{m}\left[1+\left\|g_{i_{1} \ldots i_{k}}(x)\right\|^{2}\right]\right],
$$


or the function

$\alpha(x)=\frac{\beta}{1+\sum_{k=2}^{l}\left[\sum_{i_{1}=1}^{m} \ldots \sum_{i_{k}=1}^{m}\left[1+L_{g_{i_{1}} \ldots g_{i_{k}}} V(x)\right]^{2}\right]}$ for any $0<\beta<1$.

\subsection{Controller Design}

For system (3.5.6),

$$
\begin{aligned}
& f_{0}(\bar{x})=f(\bar{x}, 0)=\left[\begin{array}{l}
\frac{1}{L_{1}}\left(\bar{x}_{2}-\bar{x}_{4}\right) \\
\frac{1}{C_{1}}\left(-\bar{x}_{1}-\frac{1}{R_{p}} \bar{x}_{2}-I_{0} e^{a x_{2}^{\star}}\left(e^{a \bar{x}_{2}}-1\right)\right) \\
\frac{1}{L_{2}}\left(-r \bar{x}_{3}-\bar{x}_{4}\right) \\
\frac{1}{C_{2}}\left(\bar{x}_{1}-\frac{1}{R_{1}} \bar{x}_{4}-\bar{x}_{5}\right. \\
\frac{1}{L_{3}}\left(\bar{x}_{4}-R_{L} \bar{x}_{5}-\bar{x}_{6}\right) \\
\frac{1}{C_{3}}\left(\bar{x}_{5}-\frac{\bar{x}_{6}}{R_{2}}\right)
\end{array}\right] \\
& g_{0}(\bar{x})=\frac{\partial f}{\partial u}(\bar{x}, 0) \\
& =\left[\begin{array}{ccc}
\frac{1}{L_{1}}\left(\bar{x}_{4}+x_{4}^{\star}\right) & 0 & 0 \\
0 & 0 & 0 \\
0 & -\frac{1}{L_{2}}\left(\bar{x}_{4}+x_{4}^{\star}\right) & 0 \\
-\frac{1}{C_{2}}\left(\bar{x}_{1}+x_{1}^{\star}\right) & \frac{1}{C_{2}}\left(\bar{x}_{3}+x_{3}^{\star}\right) & \frac{1}{C_{2}}\left(\bar{x}_{5}+x_{5}^{\star}\right) \\
0 & 0 & \frac{1}{C_{3}}\left(2 R_{L}\left(\bar{x}_{5}+x_{5}^{\star}\right)-\left(\bar{x}_{4}+x_{4}^{\star}\right)\right) \\
0 & 0 & 0
\end{array}\right]
\end{aligned}
$$




$$
\begin{aligned}
& g_{33}(\bar{x})=\left[\begin{array}{c}
0 \\
0 \\
0 \\
0 \\
-\frac{R_{L}}{L_{3}}\left(\bar{x}_{5}+x_{5}^{\star}\right) \\
0
\end{array}\right] \\
& \dot{\bar{x}}=f_{0}(\bar{x})+g_{0}(\bar{x}) u+g_{33}(\bar{x}) u_{3}^{2} \\
& L_{g_{0}} V(\bar{x})=\frac{\partial V}{\partial \bar{x}} g_{0}(\bar{x})=\left[\begin{array}{llllll}
L_{1} \bar{x}_{1} & C_{1} \bar{x}_{2} & L_{2} \bar{x}_{3} & C_{2} \bar{x}_{4} & L_{3} \bar{x}_{5} & C_{3} \bar{x}_{6}
\end{array}\right] g_{0}(\bar{x}) \\
& \left(L_{g_{0}} V(\bar{x})\right)^{T}=\left[\begin{array}{c}
x_{4}^{\star} \bar{x}_{1}-x_{1}^{\star} \bar{x}_{4} \\
-x_{4}^{\star} \bar{x}_{3}+x_{3}^{\star} \bar{x}_{4} \\
x_{5}^{\star} \bar{x}_{4}-x_{4}^{\star} \bar{x}_{5}+2 R_{L}\left(\bar{x}_{5}+x_{5}^{\star}\right) \bar{x}_{5}
\end{array}\right]
\end{aligned}
$$

It has been shown that system (3.7) is Lyapunov stable without control, which means $L_{f_{0}} V(\bar{x}) \leqslant 0 \quad \forall x_{0} \in \mathbb{R}^{n}$. Therefore, H4.1 is satisfied.

Let $u=0, y=\left(L_{g_{0}} V(\bar{x})\right)^{T}=0$.

$$
\begin{gathered}
x_{4}^{\star} \bar{x}_{1}-x_{1}^{\star} \bar{x}_{4}=0 \\
-x_{4}^{\star} \bar{x}_{3}+x_{3}^{\star} \bar{x}_{4}=0 \\
x_{5}^{\star} \bar{x}_{4}-x_{4}^{\star} \bar{x}_{5}+2 R_{L}\left(\bar{x}_{5}+x_{5}^{\star}\right) \bar{x}_{5}=0 \\
\bar{x}_{4}=\frac{x_{4}^{\star}}{x_{1}^{\star}} \bar{x}_{1} \\
\bar{x}_{4}=\frac{x_{4}^{\star}}{x_{3}^{\star}} \bar{x}_{3}
\end{gathered}
$$




$$
\dot{\bar{x}}_{3}=\frac{1}{L_{2}}\left(-\frac{x_{4}^{\star}}{x_{3}^{\star}} \bar{x}_{3}\right)
$$

Because $x_{3}^{\star}>0, x_{4}^{\star}>0, \bar{x}_{3}(t)$ is asymptotically stable. Thus, $\bar{x}_{3} \rightarrow 0$ as $t \rightarrow \infty$. Assume that $\bar{x}_{3}=0$ when $t>T$.

$$
\begin{gathered}
\bar{x}_{4}=\frac{x_{4}^{\star}}{x_{3}^{\star}} \bar{x}_{3} \quad \bar{x}_{4}=0 \quad \text { as } t>T \\
\bar{x}_{4}=\frac{x_{4}^{\star}}{x_{1}^{\star}} \bar{x}_{1} \quad \bar{x}_{1}=0 \text { as } t>T \\
\dot{\bar{x}}_{2}=\frac{1}{C_{1}}\left(-\bar{x}_{1}-\frac{\bar{x}_{2}}{R_{p}}-I_{0} e^{a x_{2}^{\star}}\left(e^{a \bar{x}_{2}}-1\right)\right) \\
=\frac{1}{C_{1}}\left(-\frac{\bar{x}_{2}}{R_{p}}-I_{0} e^{a x_{2}^{\star}}\left(e^{a \bar{x}_{2}}-1\right)\right) \text { as } t>T
\end{gathered}
$$

Using Lyapunov function

$$
V_{1}\left(\bar{x}_{2}\right)=\frac{C_{1}}{2} \bar{x}_{2}^{2}
$$

it's easy to show that $\bar{x}_{2} \rightarrow 0$ as $t \rightarrow \infty$.

For the same reason, when $t>T$,

$$
\begin{aligned}
& \dot{\bar{x}}_{5}=\frac{1}{L_{3}}\left(-R_{L} \bar{x}_{5}-\bar{x}_{6}\right) \\
& \dot{\bar{x}}_{6}=\frac{1}{C_{3}}\left(\bar{x}_{5}-\frac{\bar{x}_{6}}{R_{2}}\right)
\end{aligned}
$$

Choosing Lyapunov function

$$
V_{2}\left(\bar{x}_{5}, \bar{x}_{6}\right)=\frac{L_{3}}{2} \bar{x}_{5}^{2}+\frac{C_{3}}{2} \bar{x}_{6}^{2},
$$

one can get the conclusion that $\bar{x}_{5} \rightarrow 0, \bar{x}_{6} \rightarrow 0$ as $t \rightarrow \infty$. 
Therefore, $\lim _{t \rightarrow \infty} \bar{x}(t)=0$, H4.2 is satisfied and the theorem can be used. The designed controller is

$$
u(\bar{x})=-\alpha(\bar{x}) \frac{\left[L_{g_{0}} V(\bar{x})\right]^{T}}{1+\left\|L_{g_{0}} V(\bar{x})\right\|^{2}}
$$

where $\alpha(\bar{x})$ is the function

$$
\alpha(\bar{x})=\frac{\frac{\beta}{3}}{1+\left\|\frac{\partial V}{\partial \bar{x}}\right\|^{2} \rho^{2}(\bar{x})} \text { for any } 0<\beta<1,
$$

with

$$
\rho(\bar{x}) \geqslant 1+\left\|g_{33}(\bar{x})\right\|^{2} .
$$

Remark: Through the passivity-based design, the system is globally asymptotically stable. The controller $u$ can be arbitrarily small. However, the controller is using state feedback, which may require some states that are unmeasurable. It's possible to get output feedback controller to globally stabilize the system if the unforced system has linear property, but it's hard for general nonlinear systems. 


\section{Output Regulation and Controller Design}

In this chapter, how to design a controller to achieve the control objectives is going to be discussed. The control objectives are $v_{1} \longrightarrow v_{1}^{\star}, v_{2} \longrightarrow v_{2}^{\star}, v_{3} \longrightarrow v_{3}^{\star}$ which is a tracking or output regulation problem. First, the traditional control method will be looked into. Then, the output regulator theory will be introduced to design the controller both using state feedback and measurement feedback. However, the solution using output regulator theory is only local result. Finally, the passivity-based design is applied.

\subsection{Traditional Method for Tracking Problem}

Following the traditional method for tracking problem, another translation is needed to move the tracking objective point to origin. $\bar{x}_{2}, \bar{x}_{4}, \bar{x}_{6}$ are the signals that needed regulation. Therefore, we move the tracking point $\bar{x}_{2}=v_{1}^{\star}, \bar{x}_{4}=v_{2}^{\star}, \bar{x}_{6}=v_{3}^{\star}$ to the origin. The values of $\bar{x}_{1}, \bar{x}_{3}, \bar{x}_{5}$ are not required so they only need to be bounded. 
Let $\tilde{x}_{1}=\bar{x}_{1}, \tilde{x}_{2}=\bar{x}_{2}-v_{1}^{\star}+x_{2}^{\star}, \tilde{x}_{3}=\bar{x}_{3}, \tilde{x}_{4}=\bar{x}_{4}-v_{2}^{\star}+x_{4}^{\star}, \tilde{x}_{5}=\bar{x}_{5}, \tilde{x}_{6}=\bar{x}_{6}-v_{3}^{\star}+x_{6}^{\star}$. The system after translation is

$$
f(\tilde{x}, u)=\left[\begin{array}{l}
\frac{1}{L_{1}}\left(\tilde{x}_{2}+v_{1}^{\star}-x_{2}^{\star}-\tilde{x}_{4}-v_{2}^{\star}+x_{4}^{\star}+\left(\tilde{x}_{4}+v_{2}^{\star}\right) u_{1}\right) \\
\frac{1}{C_{1}}\left(-\tilde{x}_{1}-\frac{1}{R_{p}} \tilde{x}_{2}-\frac{1}{R_{p}}\left(v_{1}^{\star}-x_{2}^{\star}\right)-I_{0} e^{a v_{1}^{\star}}\left(e^{a \tilde{x}_{2}}-1\right)\right) \\
\frac{1}{L_{2}}\left(-r \tilde{x}_{3}-\left(\tilde{x}_{4}+v_{2}^{\star}\right) u_{2}\right) \\
\frac{1}{C_{2}}\left(\tilde{x}_{1}-\frac{1}{R_{1}} \tilde{x}_{4}-\frac{1}{R_{1}}\left(v_{2}^{\star}-x_{4}^{\star}\right)-\tilde{x}_{5}-\left(\tilde{x}_{1}+x_{1}^{\star}\right) u_{1}\right. \\
\left.\quad+\left(\tilde{x}_{3}+x_{3}^{\star}\right) u_{2}+\left(\tilde{x}_{5}+x_{5}^{\star}\right) u_{3}\right) \\
\frac{1}{L_{3}}\left(\tilde{x}_{4}+v_{2}^{\star}-x_{4}^{\star}-R_{L} \tilde{x}_{5}-\tilde{x}_{6}-v_{3}^{\star}+x_{6}^{\star}+\left(2 R_{L}\left(\tilde{x}_{5}+x_{5}^{\star}\right)\right.\right. \\
\left.\left.\quad-\left(\tilde{x}_{4}+v_{2}^{\star}\right)\right) u_{3}-R_{L}\left(\tilde{x}_{5}+x_{5}^{\star}\right) u_{3}^{2}\right) \\
\frac{1}{C_{3}}\left(\tilde{x}_{5}-\frac{1}{R_{2}} \tilde{x}_{6}\right)
\end{array}\right]
$$

To achieve the control objectives, the straight thinking is to find a controller that the system under control is stable in the sense of Lyapunov. At the same time, $\tilde{x}_{2}, \tilde{x}_{4}, \tilde{x}_{6}$ need turn to 0 as $t$ goes to $\infty$. However, due to that the system is non-affine nonlinear system, it is hard to find such a control law directly.

On the other hand, one might think about another aspect which is the physical meaning of the original system. The original system is a circuit system, states $x_{1}, x_{3}, x_{5}$ are currents, states $x_{2}, x_{4}, x_{6}$ are voltages. The control objective is the voltage regulation. When the voltages change, the currents will change correspondingly. At steady state, the voltages will track the set point, and the currents must satisfy the Kirchhoff laws. Therefore, one can calculate what values of the currents should be at the steady state. 
The original system is

$$
\begin{aligned}
& \dot{x}_{1}=\frac{1}{L_{1}}\left[x_{2}-x_{4}\left(1-u_{1}\right)\right] \\
& \dot{x}_{2}=\frac{1}{C_{1}}\left[I_{p h}-I_{0}\left(e^{a x_{2}}-1\right)-\frac{1}{R_{p}} x_{2}-x_{1}\right] \\
& \dot{x}_{3}=\frac{1}{L_{2}}\left[E-r x_{3}-x_{4} u_{2}\right] \\
& \dot{x}_{4}=\frac{1}{C_{2}}\left[-\frac{1}{R_{1}} x_{4}+x_{1}\left(1-u_{1}\right)+x_{3} u_{2}-x_{5}\left(1-u_{3}\right)\right] \\
& \dot{x}_{5}=\frac{1}{L_{3}}\left[-x_{6}+x_{4}\left(1-u_{3}\right)-R_{L} x_{5}\left(1-u_{3}\right)^{2}\right] \\
& \dot{x}_{6}=\frac{1}{C_{3}}\left[x_{5}-\frac{1}{R_{2}} x_{6}\right]
\end{aligned}
$$

which is exactly the Kirchhoff laws.

Let $x_{2}=v_{1}^{\star}, x_{4}=v_{2}^{\star}, x_{6}=v_{3}^{\star}, \dot{x}=0$, one can calculate the current $x_{1}^{\star \star}, x_{3}^{\star \star}, x_{5}^{\star \star}$ and the control signal at the steady state $u^{\star}$ by solving the equations below.

$$
\begin{aligned}
& 0=v_{1}^{\star}-v_{2}^{\star}\left(1-u_{1}^{\star}\right) \\
& 0=I_{p h}-I_{0}\left(e^{a v_{1}^{\star}}-1\right)-\frac{1}{R_{p}} v_{1}^{\star}-x_{1}^{\star \star} \\
& 0=E-r x_{3}^{\star \star}-v_{2}^{\star} u_{2}^{\star} \\
& 0=-\frac{1}{R_{1}} v_{2}^{\star}+x_{1}^{\star \star}\left(1-u_{1}^{\star}\right)+x_{3}^{\star \star} u_{2}^{\star}-x_{5}^{\star \star}\left(1-u_{3}^{\star}\right) \\
& 0=-v_{3}^{\star}+v_{2}^{\star}\left(1-u_{3}^{\star}\right)-R_{L} x_{5}^{\star \star}\left(1-u_{3}^{\star}\right)^{2} \\
& 0=x_{5}^{\star \star}-\frac{1}{R_{2}} v_{3}^{\star}
\end{aligned}
$$


Next, a translation is needed to move the tracking objective point to the origin. Let

$$
\begin{aligned}
& \hat{x}_{1}=x_{1}-x_{1}^{\star \star} \\
& \hat{x}_{2}=x_{2}-v_{1}^{\star} \\
& \hat{x}_{3}=x_{3}-x_{3}^{\star \star} \\
& \hat{x}_{4}=x_{4}-v_{2}^{\star} \\
& \hat{x}_{5}=x_{5}-x_{5}^{\star \star} \\
& \hat{x}_{6}=x_{6}-v_{3}^{\star} \\
& u_{1}=u_{1}^{\star}+\mu_{1} \\
& u_{2}=u_{2}^{\star}+\mu_{2} \\
& u_{3}=u_{3}^{\star}+\mu_{3}
\end{aligned}
$$

where $\mu(\hat{x})$ is the difference between real-time control signal $u$ and the control signal at steady state $u^{\star}$, with $\mu(0)=0$.

The system after translation is

$$
f(\hat{x}, \mu)=\left[\begin{array}{l}
\frac{1}{L_{1}}\left(\hat{x}_{2}-\left(1-u_{1}^{\star}\right) \hat{x}_{4}+\left(\hat{x}_{4}+v_{2}^{\star}\right) \mu_{1}\right) \\
\frac{1}{C_{1}}\left(-\hat{x}_{1}-\frac{1}{R_{p}} \hat{x}_{2}-I_{0} e^{a v_{1}^{\star}}\left(e^{a \hat{x}_{2}}-1\right)\right) \\
\frac{1}{L_{2}}\left(-r \hat{x}_{3}-u_{2}^{\star} \hat{x}_{4}-\left(\hat{x}_{4}+v_{2}^{\star}\right) \mu_{2}\right) \\
\frac{1}{C_{2}}\left(\left(1-u_{1}^{\star}\right) \hat{x}_{1}+u_{2}^{\star} \hat{x}_{3}-\frac{1}{R_{1}} \hat{x}_{4}-\left(1-u_{3}^{\star}\right) \hat{x}_{5}-\left(\hat{x}_{1}+x_{1}^{\star \star}\right) \mu_{1}\right. \\
\left.\quad+\left(\hat{x}_{3}+x_{3}^{\star \star}\right) \mu_{2}+\left(\hat{x}_{5}+x_{5}^{\star \star}\right) \mu_{3}\right) \\
\frac{1}{L_{3}}\left(\left(1-u_{3}^{\star}\right) \hat{x}_{4}-R_{L}\left(1+u_{3}^{\star}\right)^{2} \hat{x}_{5}-\hat{x}_{6}+\left(2 R_{L}\left(1-u_{3}^{\star}\right)\left(\hat{x}_{5}+x_{5}^{\star \star}\right)\right.\right. \\
\left.\left.\quad-\left(\hat{x}_{4}+v_{2}^{\star}\right)\right) \mu_{3}-R_{L}\left(\hat{x}_{5}+x_{5}^{\star \star}\right) \mu_{3}^{2}\right) \\
\frac{1}{C_{3}}\left(\hat{x}_{5}-\frac{1}{R_{2}} \hat{x}_{6}\right)
\end{array}\right]
$$


It can be shown that the system $\dot{\hat{x}}=f(\hat{x}, \mu)$ is GAS when $\mu=0$. One can choose the Lyapunov function

$$
\begin{gathered}
V(\hat{x})=\frac{1}{2} L_{1} \hat{x}_{1}^{2}+\frac{1}{2} C_{1} \hat{x}_{2}^{2}+\frac{1}{2} L_{2} \hat{x}_{3}^{2}+\frac{1}{2} C_{2} \hat{x}_{4}^{2}+\frac{1}{2} L_{3} \hat{x}_{5}^{2}+\frac{1}{2} C_{3} \hat{x}_{6}^{2} \\
\dot{V}(\hat{x})=-\frac{1}{R_{p}} \hat{x}_{2}^{2}-I_{0} e^{a v_{1}^{\star}}\left(e^{a \hat{x}_{2}}-1\right) \hat{x}_{2}-r \hat{x}_{3}^{2}-\frac{1}{R_{1}} \hat{x}_{4}^{2}-R_{L} \hat{x}_{5}^{2}-\frac{1}{R_{2}} \hat{x}_{6}^{2}
\end{gathered}
$$

Since $\dot{V}$ is nonpositive, system without control is stable in the sense of Lyapunov. By La Salle's Invariance Principle,

$$
\dot{V}(\hat{x})=0 \rightarrow \hat{x}_{2}=\hat{x}_{3}=\hat{x}_{4}=\hat{x}_{5}=\hat{x}_{6}=0 \rightarrow \dot{\hat{x}}_{2}=0 \rightarrow \hat{x}_{1}=0
$$

Therefore, system without control is GAS. For the original system, using constant control $u=u^{\star}$ will achieve the control objectives.

Even though the system with constant control will achieve the voltage regulation, the state signal may not have satisfied performance, which will be shown in the simulation result. In this case, $\mu$ signal needs to be designed in order to change the dynamic performance.

\subsection{Nonlinear Regulator Theory and Controller Design}

Nonlinear regulator theory is an effective method for regulating. Using this method, one can verify the result above and design the controller for improving the performance. In this section, we first review nonlinear output regulation theory and then design the controller both using state feedback and output feedback. The details and proof of nonlinear regulator theory, please see (Byrnes, 1997).

We consider a multivariable nonlinear system modeled by differential equations of the form: 


$$
\begin{aligned}
\dot{x} & =f(x, u, w) \\
\dot{w} & =S(w) \\
e & =h(x, w)
\end{aligned}
$$

The first equation describes a plant, with state $x$, defined on a neighborhood $X$ of the origin of $R^{n}$, and input $u \in R^{m}$. The input vector $w$ consists of the disturbance signals to be rejected and the reference trajectories to be tracked. The second equation describes an autonomous system, the so-called exosystem, defined in a neighborhood $W$ of the origin of $R^{s}$, which models the class of disturbance and reference signals taken into consideration. The third equation defines the error $e \in R^{p}$ between the actual plant output and the reference signal to be tracked. There are two control objectives: 1) When $w=0$, the closed loop system is locally asymptotically stable. 2) When $w \neq 0$, the error signal will turns to zero as time goes to infinity.

To apply the output regulator theory, there's two hypotheses that must be satisfied.

H5.1: $w=0$ is the equilibrium of the exosystem, and there exists a neighborhood $\hat{W} \subset W$ of the origin with the property that each initial condition $w(0) \in \hat{W}$ is Poisson stable.

H5.2: The pair $(A, B)=\left(\left.\frac{\partial f}{\partial x}\right|_{(x, u, w)=(0,0,0)},\left.\frac{\partial f}{\partial u}\right|_{(x, u, w)=(0,0,0)}\right)$ has a stabilizable linear approximation at $x=0$.

The state feedback regulator problem is solvable if and only if there exist $C^{k}(k \geqslant$ 2) mappings $x=\pi(w)$, with $\pi(0)=0$, and $u=c(w)$, with $c(0)=0$, both defined in a neighborhood $W^{0} \subset W$ of 0 , satisfying the conditions 


$$
\begin{array}{r}
f(\pi(w), c(w), w)=0 \\
h(\pi(w), w)=0
\end{array}
$$

The designed controller is

$$
u=c(w)+K(x-\pi(w))
$$

where $K$ is any matrix which places the eigenvalues of $A+B K$ in $C^{-}$.

Remark: The result of output regulator theory is local result defined in the neighborhood of $w=0$ since it uses linear approximation. The benefit of the output regulator theory is that one can manage the dynamic performance by choosing certain matrix $K$. It is the same strategy as pole assignment in the linear system.

In this study, there is no disturbance. The reference signals to be followed are the voltages. That is, $w=\left[w_{1}, w_{2}, w_{3}\right]^{T}=\left[\bar{x}_{2}^{\star}, \bar{x}_{4}^{\star}, \bar{x}_{6}^{\star}\right]=\left[v_{1}^{\star}-x_{2}^{\star}, v_{2}^{\star}-x_{4}^{\star}, v_{3}^{\star}-x_{6}^{\star}\right]^{T}$. As a result, $S(w)=0$ in this paper.

We can rewrite our model into the standard form of output regulator theory.

$$
\begin{aligned}
\dot{\bar{x}} & =f(\bar{x}, u)+p(\bar{x}) w \\
\dot{w} & =S(w)=0 \\
e & =h(\bar{x})+q(w)
\end{aligned}
$$


where

$$
f(\bar{x}, u)=\left[\begin{array}{c}
\frac{1}{L_{1}}\left(\bar{x}_{2}-\bar{x}_{4}+\left(\bar{x}_{4}+x_{4}^{\star}\right) u_{1}\right) \\
\frac{1}{C_{1}}\left(-\bar{x}_{1}-\frac{\bar{x}_{2}}{R_{p}}-I_{0} e^{a x_{2}^{\star}}\left(e^{a \bar{x}_{2}}-1\right)\right) \\
\frac{1}{L_{2}}\left(-r \bar{x}_{3}-\left(\bar{x}_{4}+x_{4}^{\star}\right) u_{2}\right) \\
\frac{1}{C_{2}}\left(\bar{x}_{1}-\frac{\bar{x}_{4}}{R_{1}}-\bar{x}_{5}-\left(\bar{x}_{1}+x_{1}^{\star}\right) u_{1}\right. \\
\left.+\left(\bar{x}_{3}+x_{3}^{\star}\right) u_{2}+\left(\bar{x}_{5}+x_{5}^{\star}\right) u_{3}\right) \\
\frac{1}{L_{3}}\left(\bar{x}_{4}-R_{L} \bar{x}_{5}-\bar{x}_{6}+\left(2 R_{L}\left(\bar{x}_{5}+x_{5}^{\star}\right)\right.\right. \\
\left.\left.-\left(\bar{x}_{4}+x_{4}^{\star}\right)\right) u_{3}-R_{L}\left(\bar{x}_{5}+x_{5}^{\star}\right) u_{3}^{2}\right) \\
\frac{1}{C_{3}}\left(\bar{x}_{5}-\frac{\bar{x}_{6}}{R_{2}}\right)
\end{array}\right]
$$

$$
p(\bar{x})=0, h(\bar{x})=\left[\begin{array}{c}
\bar{x}_{2} \\
\bar{x}_{4} \\
\bar{x}_{6}
\end{array}\right], q(w)=\left[\begin{array}{c}
-w_{1} \\
-w_{2} \\
-w_{3}
\end{array}\right]
$$

H5.1 is stisfied when $w$ is constant. The linear approximation at $\bar{x}=0$ is shown below.

$$
A=\left[\begin{array}{cccccc}
0 & \frac{1}{L_{1}} & 0 & -\frac{1}{L_{1}} & 0 & 0 \\
-\frac{1}{C_{1}} & -\frac{1}{R_{p} C_{1}}-a I_{0} e^{a x_{2}^{\star}} & 0 & 0 & 0 & 0 \\
0 & 0 & -\frac{r}{L_{2}} & 0 & 0 & 0 \\
\frac{1}{C_{2}} & 0 & 0 & -\frac{1}{R_{1} C_{2}} & -\frac{1}{C_{2}} & 0 \\
0 & 0 & 0 & \frac{1}{L_{3}} & -\frac{R_{L}}{L_{3}} & -\frac{1}{L_{3}} \\
0 & 0 & 0 & 0 & \frac{1}{C_{3}} & -\frac{1}{C_{3} R_{2}}
\end{array}\right]
$$


$B=\left[\begin{array}{ccc}\frac{1}{L_{1}} x_{4}^{\star} & 0 & 0 \\ 0 & 0 & 0 \\ 0 & -\frac{r}{L_{2}} x_{4}^{\star} & 0 \\ -\frac{1}{C_{2}} x_{1}^{\star} & \frac{1}{C_{2}} x_{3}^{\star} & \frac{1}{C_{2}} x_{5}^{\star} \\ 0 & 0 & \frac{1}{L_{3}}\left(2 R_{L} x_{5}^{\star}-x_{4}^{\star}\right) \\ 0 & 0 & 0\end{array}\right]$ which is stabilizable. Applying the theory to our model, one can get

$$
\begin{aligned}
& \pi_{2}(w)-\pi_{4}(w)+\left(\pi_{4}(w)+x_{4}^{\star}\right) c_{1}(w)=0 \\
& -\pi_{1}(w)-\frac{\pi_{2}(w)}{R_{p}}-I_{0} e^{a x_{2}^{\star}}\left(e^{a \pi_{2}(w)}-1\right)=0 \\
& -r \pi_{3}(w)-\left(\pi_{4}(w)+x_{4}^{\star}\right) c_{2}(w)=0 \\
& \pi_{1}(w)-\frac{\pi_{4}(w)}{R_{1}}-\pi_{5}(w)-\left(\pi_{1}(w)+x_{1}^{\star}\right) c_{1}(w) \\
& \quad+\left(\pi_{3}(w)+x_{3}^{\star}\right) c_{2}(w)+\left(\pi_{5}(w)+x_{5}^{\star}\right) c_{3}(w)=0 \\
& \left.\quad-\left(\pi_{4}(w)+x_{4}^{\star}\right)\right) c_{3}(w)-R_{L}\left(\pi_{5}(w)+x_{5}^{\star}\right) c_{3}^{2}(w)=0 \\
& \pi_{5}(w)-\frac{\pi_{6}(w)}{R_{2}}=0 \\
& \pi_{2}(w)=R_{1}, \quad \pi_{5}(w)-\pi_{6}(w)+\left(2 R_{L}\left(\pi_{5}(w)+x_{5}^{\star}\right)\right.
\end{aligned}
$$


After solving the equations, one can get

$$
\begin{aligned}
& \pi_{1}(w)=-\frac{1}{R_{p}} w_{1}-I_{0} e^{a x_{2}^{\star}}\left(e^{a w_{1}}-1\right) \\
& \pi_{2}(w)=w_{1} \\
& \pi_{3}(w)=-\frac{w_{2}+x_{4}^{\star}}{r} c_{2}(w) \\
& \pi_{4}(w)=w_{2} \\
& \pi_{5}(w)=\frac{1}{R_{2}} w_{3} \\
& \pi_{6}(w)=w_{3} \\
& c_{1}(w)=\frac{w_{2}-w_{1}}{w_{2}+x_{4}^{\star}} \\
& -\frac{1}{R_{p}} w_{1}-I_{0} e^{a x_{2}^{\star}}\left(e^{a w_{1}}-1\right)-\frac{w_{2}}{R_{1}}-\frac{1}{R_{2}} w_{3} \\
& \quad-\left(-\frac{1}{R_{p}} w_{1}-I_{0} e^{a x_{2}^{\star}}\left(e^{a w_{1}}-1\right)+x_{1}^{\star}\right) \frac{w_{2}-w_{1}}{w_{2}+x_{4}^{\star}} \\
& \quad+\left(-\frac{w_{2}+x_{4}^{\star}}{r} c_{2}(w)+x_{3}^{\star}\right) c_{2}(w)+\left(\frac{1}{R_{2}} w_{3}+x_{5}^{\star}\right) c_{3}(w)=0 \\
& w_{2}-R_{L} \frac{1}{R_{2}} w_{3}-w_{3}+\left(2 R_{L}\left(\frac{1}{R_{2}} w_{3}+x_{5}^{\star}\right)\right. \\
& \left.\quad-\left(w_{2}+x_{4}^{\star}\right)\right) c_{3}(w)-R_{L}\left(\frac{1}{R_{2}} w_{3}+x_{5}^{\star}\right) c_{3}^{2}(w)=0
\end{aligned}
$$

Finally, the control law is $u=c(w)+K(x-\pi(w))$, where $K$ is any matrix which places the eigenvalues of $A+B K$ in $C^{-}$. Due to the restriction of $u, 0<u<1$. 
Remark: Equation (5.1.2) and (5.2.13) are same equation since

$$
\begin{aligned}
\pi_{1}(w)+x_{1}^{\star} & =x_{1}^{\star \star} \\
\pi_{2}(w)+x_{2}^{\star} & =v_{1}^{\star} \\
\pi_{3}(w)+x_{3}^{\star} & =x_{3}^{\star \star} \\
\pi_{4}(w)+x_{4}^{\star} & =v_{2}^{\star} \\
\pi_{5}(w)+x_{5}^{\star} & =x_{5}^{\star \star} \\
\pi_{6}(w)+x_{6}^{\star} & =v_{3}^{\star} \\
c_{1}(w) & =u_{1}^{\star} \\
c_{2}(w) & =u_{2}^{\star} \\
c_{3}(w) & =u_{3}^{\star} \\
K(x-\pi(w)) & =\mu
\end{aligned}
$$

This verifies the result of the previous section.

In practical problem, sometimes the full state feedback controller is not implementable because some of the state is not measurable. In this case, one could use partial state feedback control.

Partial state feedback control means the control signal only use the states that are measurable. By choosing certain $K$ matrix, one can let the unmeasurable state do not appear in the control. For example, if state $x_{1}$ is not measurable, choose the first column of $K$ to be zero so that state $x_{1}$ will not appear in $u$. However, in order to promise the stability, all the eigenvalues of $A+B K$ must set in $C^{-}$. 
However, if partial state feedback doesn't work, one could use measurement feedback control. Since the signals to be tracking are known constants, there's no need to build observers for $w$. The strategy of the measurement feedback design is the same as the linear case.

For a stabilizable and detectable linear system

$$
\begin{aligned}
\dot{x} & =A x+B u \\
y & =C x
\end{aligned}
$$

there exist a measurement feedback controller of the form

$$
\begin{aligned}
& \dot{\xi}=F \xi+G y \\
& u=H \xi
\end{aligned}
$$

such that the closed-loop system is AS where $F=A+B K-M C, H=K, G=M$ and the eigenvalues of $A+B K$ and $A-M C$ must set in $C^{-}$. 
In order to apply the measurement feedback design, the first step is to do the translation and move the system to the objective point. The system after translation is

$$
\begin{aligned}
& f(\hat{x}, \mu)= \\
& {\left[\begin{array}{l}
\frac{1}{L_{1}}\left(\hat{x}_{2}-\left(1-c_{1}(w)\right) \hat{x}_{4}+\left(\hat{x}_{4}+\pi_{4}(w)+x_{4}^{\star}\right) \mu_{1}\right) \\
\frac{1}{C_{1}}\left(-\hat{x}_{1}-\frac{1}{R_{p}} \hat{x}_{2}-I_{0} e^{a\left(\pi_{2}(w)+x_{2}^{\star}\right)}\left(e^{a \hat{x}_{2}}-1\right)\right) \\
\frac{1}{L_{2}}\left(-r \hat{x}_{3}-c_{2}(w) \hat{x}_{4}-\left(\hat{x}_{4}+\pi_{4}(w)+x_{4}^{\star}\right) \mu_{2}\right) \\
\frac{1}{C_{2}}\left(\left(1-c_{1}(w)\right) \hat{x}_{1}+c_{2}(w) \hat{x}_{3}-\frac{1}{R_{1}} \hat{x}_{4}-\left(1-c_{3}(w)\right) \hat{x}_{5}\right. \\
\left.\quad-\left(\hat{x}_{1}+\pi_{1}(w)+x_{1}^{\star}\right) \mu_{1}+\left(\hat{x}_{3}+\pi_{3}(w)+x_{3}^{\star}\right) \mu_{2}+\left(\hat{x}_{5}+\pi_{5}(w)+x_{5}^{\star}\right) \mu_{3}\right) \\
\frac{1}{L_{3}}\left(\left(1-c_{3}(w)\right) \hat{x}_{4}-R_{L}\left(1+c_{3}(w)\right)^{2} \hat{x}_{5}-\hat{x}_{6}+\left(2 R _ { L } ( 1 - c _ { 3 } ( w ) ) \left(\hat{x}_{5}\right.\right.\right. \\
\left.\left.\left.\quad+\pi_{5}(w)+x_{5}^{\star}\right)-\left(\hat{x}_{4}+\pi_{4}(w)+x_{4}^{\star}\right)\right) \mu_{3}-R_{L}\left(\hat{x}_{5}+\pi_{5}(w)+x_{5}^{\star}\right) \mu_{3}^{2}\right) \\
\frac{1}{C_{3}}\left(\hat{x}_{5}-\frac{1}{R_{2}} \hat{x}_{6}\right)
\end{array}\right.}
\end{aligned}
$$

$$
y=\left[\begin{array}{c}
\hat{x}_{2}+v_{1}^{\star} \\
\hat{x}_{4}+v_{2}^{\star} \\
\hat{x}_{6}+v_{3}^{\star}
\end{array}\right]
$$

which is the same as the one in the previous section.

The next step is do the linear approximation.

$$
\left[\begin{array}{cccccc}
\hat{A}= & \frac{1}{L_{1}} & 0 & -\frac{1}{L_{1}}\left(1-c_{1}(w)\right) & 0 & 0 \\
0 & -\frac{1}{R_{p} C_{1}}-a I_{0} e^{a\left(\pi_{2}(w)+x_{2}^{\star}\right)} & 0 & 0 & 0 & 0 \\
-\frac{1}{C_{1}} & 0 & -\frac{r}{L_{2}} & -\frac{1}{L_{2}} c_{2}(w) & 0 & 0 \\
0 & 0 & \frac{1}{C_{2}} c_{2}(w) & -\frac{1}{R_{1} C_{2}} & -\frac{1}{C_{2}}\left(1-c_{3}(w)\right) & 0 \\
\frac{1}{C_{2}}\left(1-c_{1}(w)\right) & 0 & 0 & \frac{1}{L_{3}}\left(1-c_{3}(w)\right) & -\frac{R_{L}}{L_{3}}\left(1+c_{3}(w)\right)^{2} & -\frac{1}{L_{3}} \\
0 & 0 & 0 & 0 & \frac{1}{C_{3}} & -\frac{1}{C_{3} R_{2}}
\end{array}\right]
$$




$$
\begin{aligned}
& \hat{B}= \\
& {\left[\begin{array}{ccc}
\frac{1}{L_{1}}\left(\pi_{4}(w)+x_{4}^{\star}\right) & 0 & 0 \\
0 & 0 & 0 \\
0 & -\frac{1}{L_{2}}\left(\pi_{4}(w)+x_{4}^{\star}\right) & 0 \\
-\frac{1}{C_{2}}\left(\pi_{1}(w)+x_{1}^{\star}\right) & \frac{1}{C_{2}}\left(\pi_{3}(w)+x_{3}^{\star}\right) & \frac{1}{C_{2}}\left(\pi_{5}(w)+x_{5}^{\star}\right) \\
0 & 0 & 2 R_{L} \frac{1}{C_{3}}\left(1-c_{3}(w)\right)\left(\pi_{5}(w)+x_{5}^{\star}\right)-\frac{1}{C_{3}}\left(\pi_{4}(w)+x_{4}^{\star}\right) \\
0 & 0 & 0
\end{array}\right]} \\
& \hat{C}=\left[\begin{array}{llllll}
0 & 1 & 0 & 0 & 0 & 0 \\
0 & 0 & 0 & 1 & 0 & 0 \\
0 & 0 & 0 & 0 & 0 & 1
\end{array}\right] \\
& \operatorname{rank}\left[\hat{B} \quad \hat{A} \hat{B} \quad \hat{A}^{2} \hat{B} \quad \hat{A}^{3} \hat{B} \quad \hat{A}^{4} \hat{B} \quad \hat{A}^{5} \hat{B}\right]=6
\end{aligned}
$$

System is controllable.

$\operatorname{rank}\left[\begin{array}{c}\hat{C} \\ \hat{C} \hat{A} \\ \hat{C} \hat{A}^{2} \\ \hat{C} \hat{A}^{3} \\ \hat{C} \hat{A}^{4} \\ \hat{C} \hat{A}^{5}\end{array}\right]=6$

System is observable.

Therefore, by choosing certain matrix $K$ and $M$, one can form the measurement feedback compensator

$$
\begin{aligned}
\dot{\xi} & =F \xi+G y \\
\mu & =H \xi
\end{aligned}
$$

Due to the limitation of $u, 0 \leqslant \mu+c(w) \leqslant 1$. 


\subsection{Passivity-based Design}

Passivity-based design can also be used for set point regulation. The advantage of passivitybased design is that it provides an arbitrary small controller to globally stabilize the system. However, the controller itself is nonlinear and there's no guidance for how to choose a controller to improve the dynamic performance.

For system (5.1.4) and Lyapunov function (5.1.5), H4.1 is satisfied.

$$
\begin{aligned}
& f_{0}(\hat{x})=f(\hat{x}, 0)=\left[\begin{array}{l}
\frac{1}{L_{1}}\left(\hat{x}_{2}-\left(1-u_{1}^{\star}\right) \hat{x}_{4}\right) \\
\frac{1}{C_{1}}\left(-\hat{x}_{1}-\frac{1}{R_{p}} \hat{x}_{2}-I_{0} e^{a v_{1}^{\star}}\left(e^{a \hat{x}_{2}}-1\right)\right) \\
\frac{1}{L_{2}}\left(-r \hat{x}_{3}-u_{2}^{\star} \hat{x}_{4}\right) \\
\frac{1}{C_{2}}\left(\left(1-u_{1}^{\star}\right) \hat{x}_{1}+u_{2}^{\star} \hat{x}_{3}-\frac{1}{R_{1}} \hat{x}_{4}-\left(1-u_{3}^{\star}\right) \hat{x}_{5}\right. \\
\frac{1}{L_{3}}\left(\left(1-u_{1}^{\star}\right) \hat{x}_{4}-R_{L}\left(1+u_{1}^{\star}\right)^{2} \hat{x}_{5}-\hat{x}_{6}\right) \\
\frac{1}{C_{3}}\left(\hat{x}_{5}-\frac{\hat{x}_{6}}{R_{2}}\right)
\end{array}\right] \\
& g_{0}(\hat{x})=\frac{\partial f}{\partial \mu}(\hat{x}, 0) \\
& =\left[\begin{array}{ccc}
\frac{1}{L_{1}}\left(\hat{x}_{4}+v_{2}^{\star}\right) & 0 & 0 \\
0 & 0 & 0 \\
0 & -\frac{1}{L_{2}}\left(\hat{x}_{4}+v_{2}^{\star}\right) & 0 \\
-\frac{1}{C_{2}}\left(\hat{x}_{1}+x_{1}^{\star \star}\right) & \frac{1}{C_{2}}\left(\hat{x}_{3}+x_{3}^{\star \star}\right) & \frac{1}{C_{2}}\left(\hat{x}_{5}+x_{5}^{\star \star}\right) \\
0 & 0 & \frac{1}{C_{3}}\left(2 R_{L}\left(1-u_{3}^{\star}\right)\left(\hat{x}_{5}+x_{5}^{\star \star}\right)-\left(\hat{x}_{4}+v_{2}^{\star}\right)\right) \\
0 & 0 & 0
\end{array}\right]
\end{aligned}
$$




$$
\begin{aligned}
& g_{33}(\hat{x})=\left[\begin{array}{c}
0 \\
0 \\
0 \\
0 \\
-\frac{R_{L}}{L_{3}}\left(\hat{x}_{5}+x_{5}^{\star \star}\right) \\
0
\end{array}\right] \\
& \dot{\hat{x}}=f_{0}(\hat{x})+g_{0}(\hat{x}) \mu+g_{33}(\hat{x}) \mu_{3}^{2} \\
& L_{g_{0}} V(\hat{x})=\frac{\partial V}{\partial \hat{x}} g_{0}(\hat{x})=\left[\begin{array}{llllll}
L_{1} \hat{x}_{1} & C_{1} \hat{x}_{2} & L_{2} \hat{x}_{3} & C_{2} \hat{x}_{4} & L_{3} \hat{x}_{5} & C_{3} \hat{x}_{6}
\end{array}\right] g_{0}(\hat{x}) \\
& \left(L_{g_{0}} V(\hat{x})\right)^{T}=\left[\begin{array}{c}
v_{2}^{\star} \hat{x}_{1}-x_{1}^{\star \star} \hat{x}_{4} \\
-v_{2}^{\star} \hat{x}_{3}+x_{3}^{\star \star} \hat{x}_{4} \\
x_{5}^{\star \star} \hat{x}_{4}-v_{2}^{\star} \hat{x}_{5}+2 R_{L}\left(1-u_{3}^{\star}\right)\left(\hat{x}_{5}+x_{5}^{\star \star}\right) \hat{x}_{5}
\end{array}\right]
\end{aligned}
$$

Using the same argument in section 4.4, one can prove that system is zero-state detectable and H4.2 is satisfied.

The designed controller is

$$
\mu(\hat{x})=-\alpha(\hat{x}) \frac{\left[L_{g_{0}} V(\hat{x})\right]^{T}}{1+\left\|L_{g_{0}} V(\hat{x})\right\|^{2}}
$$

where $\alpha(\hat{x})$ is the function

$$
\alpha(\hat{x})=\frac{\frac{\beta}{3}}{1+\left\|\frac{\partial V}{\partial \hat{x}}\right\|^{2} \rho^{2}(\hat{x})} \text { for any } 0<\beta<1,
$$

with

$$
\rho(\hat{x}) \geqslant 1+\left\|g_{33}(\hat{x})\right\|^{2} .
$$


For example, when choosing $\beta=0.5$ and $\rho(\hat{x})=1+\left\|g_{33}(\hat{x})\right\|^{2}$, the designed controller is

$$
\begin{gathered}
\mu(\hat{x})=-\frac{0.5 / 3}{1+\left(L_{1}^{2} \hat{x}_{1}^{2}+C_{1}^{2} \hat{x}_{2}^{2}+L_{2}^{2} \hat{x}_{3}^{2}+C_{2}^{2} \hat{x}_{4}^{2}+L_{3}^{2} \hat{x}_{5}^{2}+C_{3}^{2} \hat{x}_{6}^{2}\right)\left(1+\left(\frac{R_{L}}{L_{3}}\left(\hat{x}_{5}+x_{5}^{\star \star}\right)^{2}\right)^{2}\right.} \\
\frac{\left[L_{g_{0}} V(\hat{x})\right]^{T}}{1+\left(\left(v_{2}^{\star} \hat{x}_{1}-x_{1}^{\star \star} \hat{x}_{4}\right)^{2}+\left(-v_{2}^{\star} \hat{x}_{3}+x_{3}^{\star \star} \hat{x}_{4}\right)^{2}+\left(x_{5}^{\star \star} \hat{x}_{4}-v_{2}^{\star} \hat{x}_{5}+2 R_{L}\left(1-u_{3}^{\star}\right)\left(\hat{x}_{5}+x_{5}^{\star \star}\right) \hat{x}_{5}\right)^{2}\right)}
\end{gathered}
$$




\section{Case Studies}

In this chapter, the simulation results of controllers discussed in the previous is shown. All the simulation results are generated by MATLAB.

There's two cases of simulation. One is the illumination change when the load remains the same. The other is the load change while the illumination remains constant.

\subsection{Illumination Change}

All the results below reflect the illumination change when the load remains the same. The situation is assumed as follows:

At $t=0 s$, system works at steady state, the PV is working at its MPPT, the photogenerated current $I_{p} h$ relates to the irradiance. At $t=0.2 s$, the irradiance decreases, so is $I_{p} h$. At $t=0.4 s$, there is no illumination, $I_{p} h$ becomes zero. At $t=0.6 s$ and $t=0.8 s$, the illumination recovers gradually.

\subsubsection{Constant Feedback Control}

Figure 6.1 shows the simulation result of the constant feedback control.

Apparently, the dynamic performance is not satisfied using constant feedback control. At $t=0.4 s$, the output voltage of PV cannot turns to zero in a short time as the light out, and an oscillation happens due to the inductor, capacitor and lack of shunt resistor 

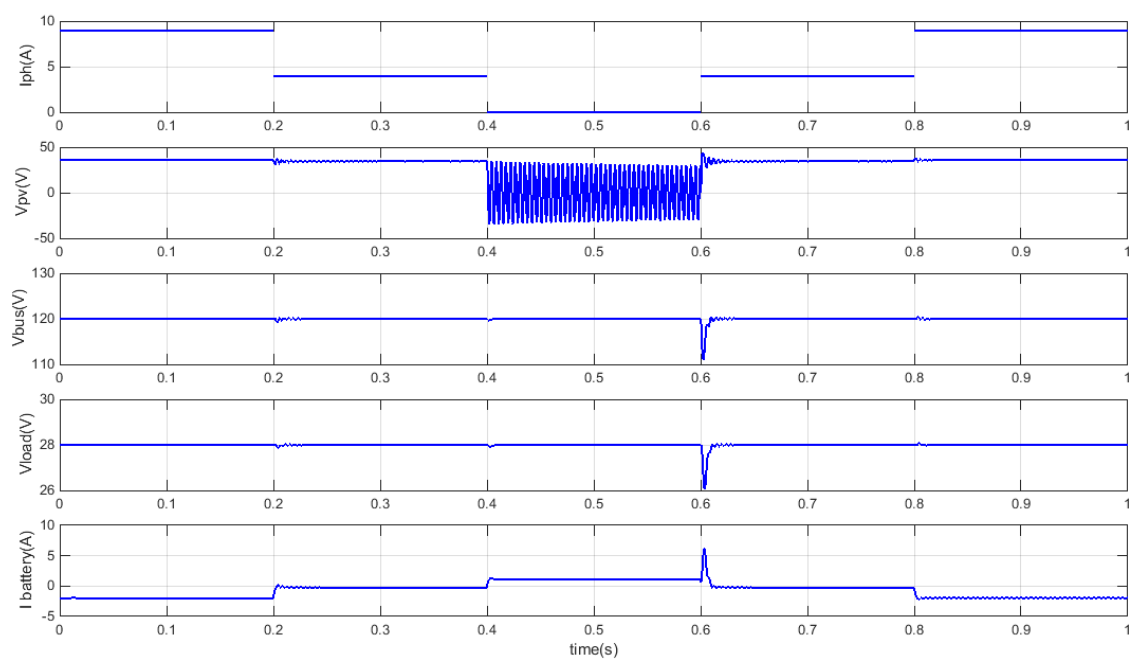

Figure 6.1. simulation result of illumination changes using constant feedback control

of the dc converter. Some applications use a series of shunt resistor to solve this oscillation problem. At $t=0.6 \mathrm{~s}$, the bus voltage has a big jump which is out of the range of safety. Therefore, dynamic control is needed to improve the dynamic performance.

\subsubsection{State Feedback Control}

Figure 6.2 shows the simulation result of the state feedback control.

The $K$ matrix is chosen as $\left[\begin{array}{cccccc}-0.01 & 0 & 0 & 0 & 0 & 0 \\ 0 & 0 & 0 & 0 & 0 & 0 \\ 0 & 0 & 0 & 0 & 0 & 0\end{array}\right]$.

It's clearly that the closed-loop system has good dynamic performance. The PV output voltage equals to the value that maximizes the power output and both bus voltage and load voltage recover in a short time without huge deviation. The current of battery is within tolerance too. 

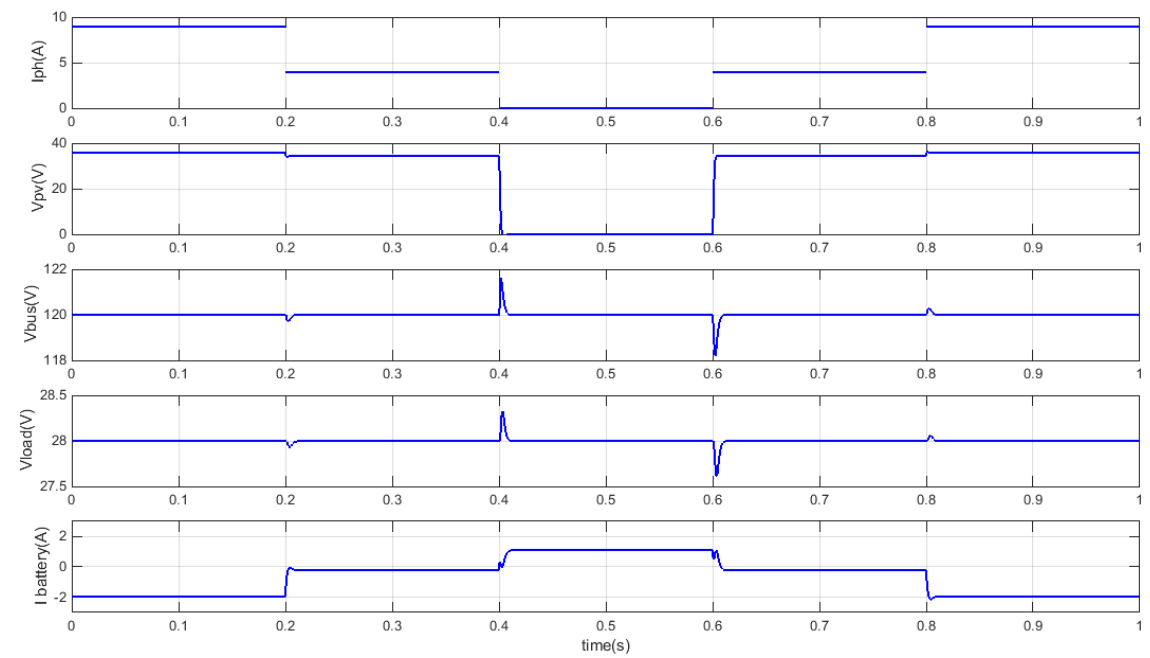

Figure 6.2. simulation result of illumination changes using state feedback control

Only one state $x_{1}$ is used for the controller design. Unfortunately, the state $x_{1}$ is an unmeasurable state. Thus, the controller is not implementable.

\subsubsection{Measurement Feedback Control}

Figure 6.3 shows the simulation result of the measurement feedback control.

The $K$ matrix is chosen as $\left[\begin{array}{cccccc}-0.01 & 0 & 0 & 0 & 0 & 0 \\ 0 & 0 & 0 & 0 & 0 & 0 \\ 0 & 0 & 0 & 0 & 0 & 0\end{array}\right]$.



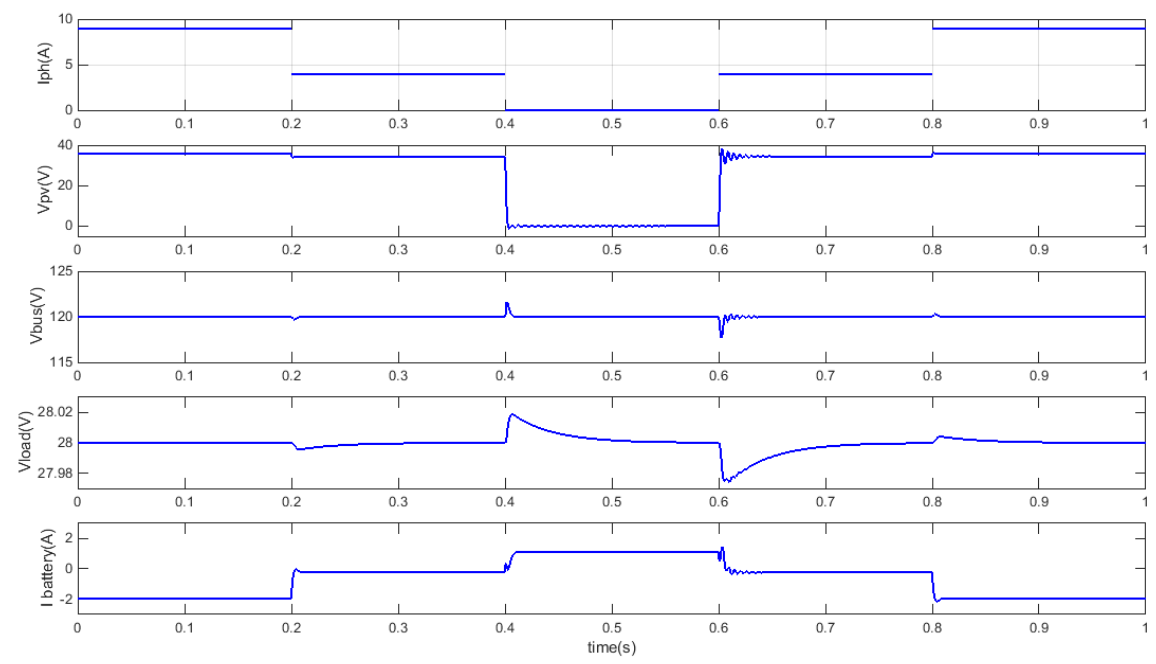

Figure 6.3. simulation result of illumination changes using measurement feedback control

As shown above, dynamic performance is a little worse than state feedback control, but it is still within tolerance. The voltages and the current recover in a short time without huge deviation.

\subsection{Load Change}

All the results below reflect the load change when the illumination remains the same. The situation is assumed as follows:

At $t=0 \mathrm{~s}$, system works at steady state, the PV is working at its maximum power output and the battery is charging. At $t=0.2 \mathrm{~s}$, the bus load increases ( $R_{1}$ decreases); At $t=0.4 s$, the load decreases ( $R_{2}$ increases).

\subsubsection{Constant Feedback Control}

Figure 6.4 shows the simulation result of the constant feedback control. 

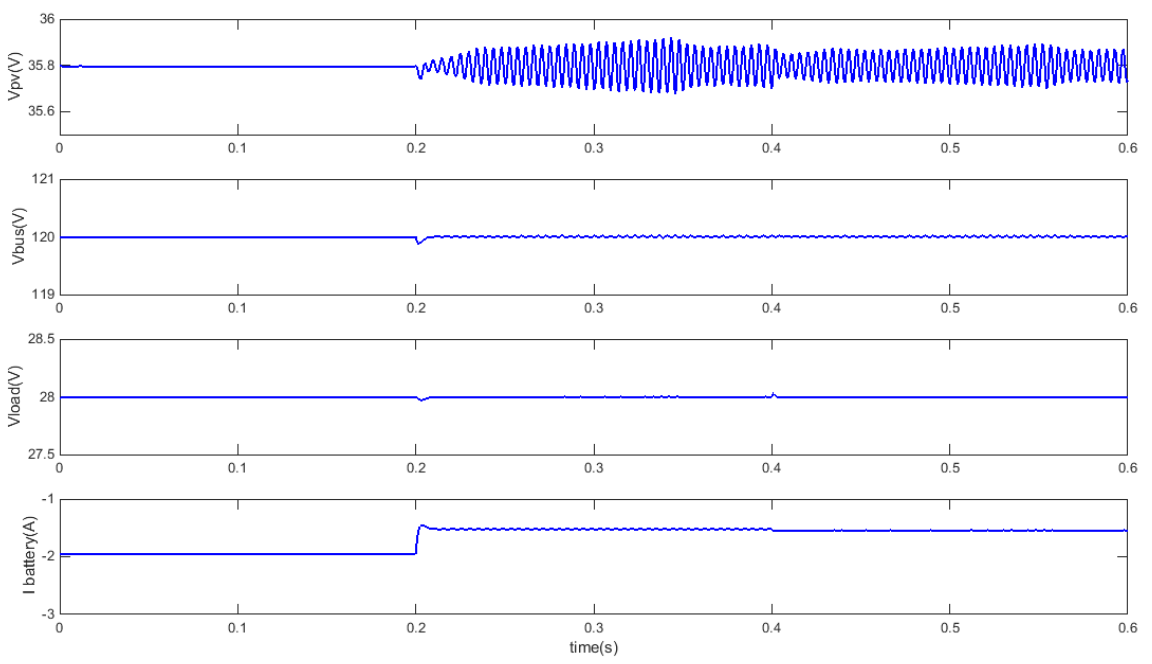

Figure 6.4. simulation result of load changes using constant feedback control

Similar to the case when illumination changes, the dynamic performance is not satisfied using constant feedback control. The output voltage of PV cannot recover in a short time as the load changes, and an oscillation happens due to the inductor, capacitor and lack of shunt resistor of the dc converter.

\subsubsection{State Feedback Control}

Figure 6.5 shows the simulation result of the state feedback control.

The $K$ matrix is chosen as $\left[\begin{array}{cccccc}-0.01 & 0 & 0 & 0 & 0 & 0 \\ 0 & 0 & 0 & 0 & 0 & 0 \\ 0 & 0 & 0 & 0 & 0 & 0\end{array}\right]$.

Apparently, the closed-loop system has good dynamic performance. The output voltage of PV remains the same and bus voltage and load voltage recover after a short time. The charging rate of the battery changes correspondingly.

Only one state $x_{1}$ is used for the controller design. Unfortunately, the state $x_{1}$ is an unmeasurable state. Thus, the controller is not implementable. 

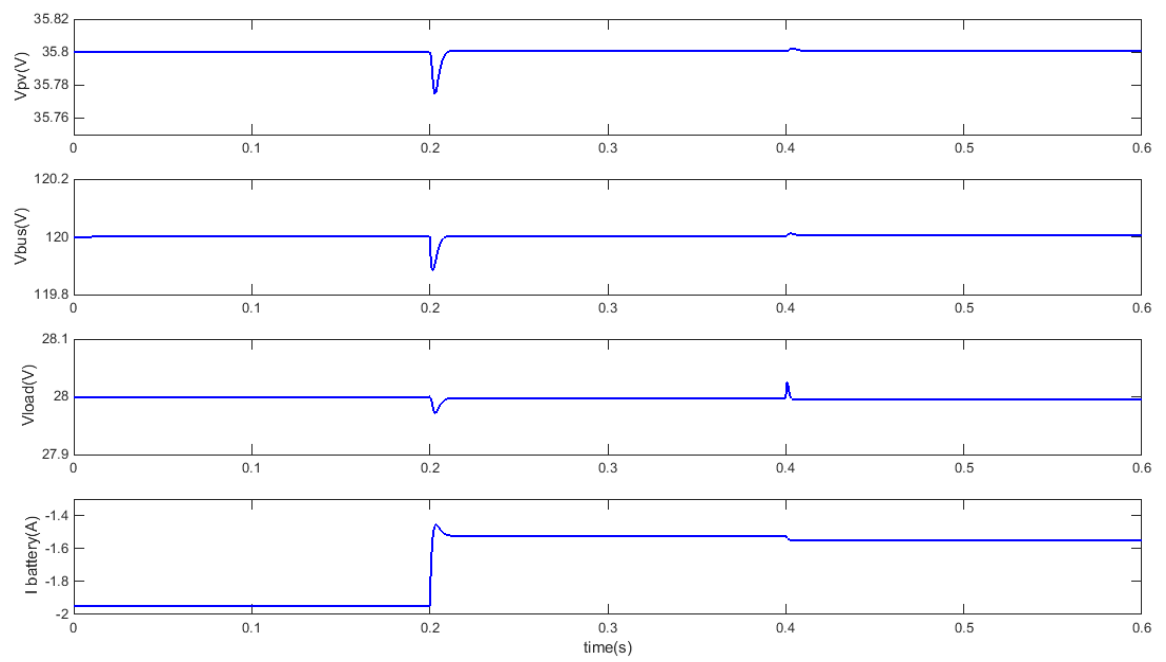

Figure 6.5. simulation result of load changes using state feedback control

\subsubsection{Measurement Feedback Control}

Figure 6.6 shows the simulation result of the measurement feedback control.
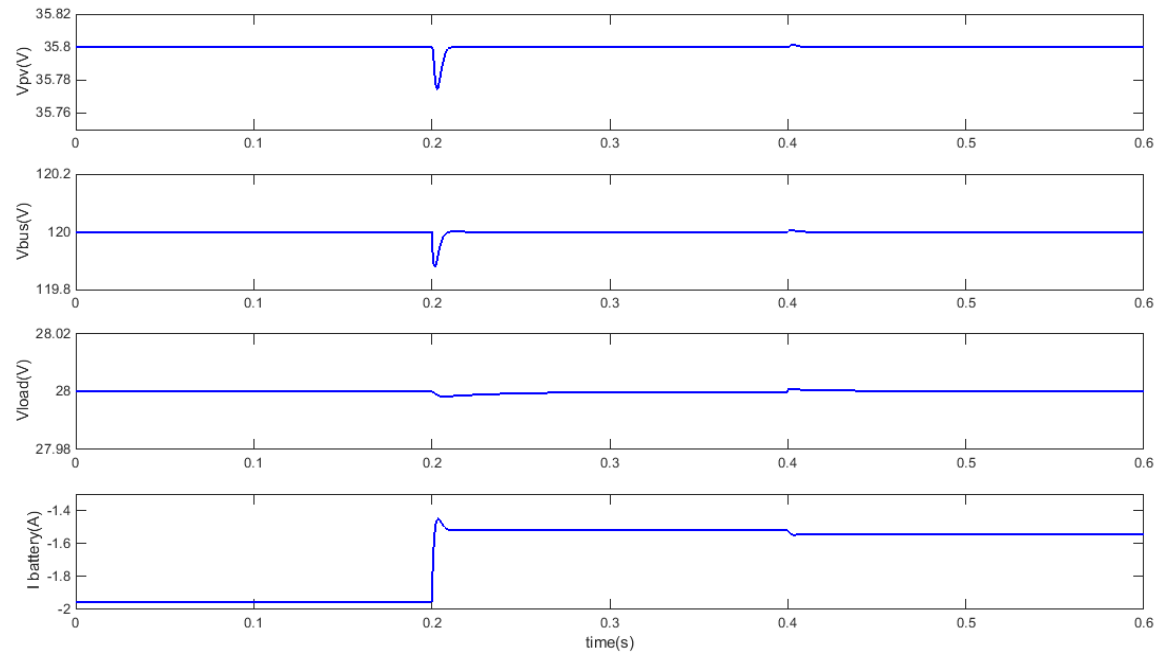

Figure 6.6. simulation result of load changes using measurement feedback control 
The $K$ matrix is chosen as $\left[\begin{array}{cccccc}-0.01 & 0 & 0 & 0 & 0 & 0 \\ 0 & 0 & 0 & 0 & 0 & 0 \\ 0 & 0 & 0 & 0 & 0 & 0\end{array}\right]$.

Using the measurement feedback control, the closed-loop system has good dynamic performance similar to the state feedback control. 


\section{Future Study}

In this section, the future study of DC-microgrid is discussed. There's three possible directions. The first one is considering more practical situation, which means adding disturbance into the system. The second direction is dealing with more complexed system, such as a DC-microgrid with multiple PVs and batteries, or even connect with AC power grid. The last one is using more advanced control method. Adaptive control or robust control may be applied to this problem.

\subsection{System with Disturbance}

In the case study, the dynamic performance of system is researched for known change of illumination and load. If the change of illumination and load is unknown, it can be modeled as disturbance. The disturbance may also appear at output due to inaccuracy of the sensor. The disturbance can be constant or periodical. For example, the inaccuracy of the sensor will cause constant disturbance and the change of illumination can be modeled as periodical disturbance. Also, the maximum power point of PV may also be unknown, which means the control objective is unknown.

The output regulator theory has the ability to deal with the problem with disturbance listed above. The input vector $w$ has two components, which are disturbance signals to 
be rejected and the reference trajectories to be tracked. Using the same technique, one can get the controller by full state feedback. However, the controller $u=c(w)+K(x-$ $\pi(w))$ may not be complementable since $w$ insists the disturbance that is unknown. There's two possible ways to solve the problem. One is using partial state feedback. By choosing suitable $K$ matrix, it is possible that all the unknown $x$ and $w$ do not appear in the controller. If the partial state feedback control fails, the other approach is the output feedback control. In this case, the observer is needed not only for unmeasurable state $x$, but also unmeasurable disturbance $w$.

\subsection{More Complexed System}

The following firgue shows the configuration of a DC-microgrid with two PVs and two batteries.

As shown in the picture, the whole system insists of two subsystem connecting with a transmission line. With multiple PVs and batteries, the system has more flexibility. If the PVs and batteries have enough capacity, one PV and one battery can supply for the whole system. In another case, if someone wants the subsystem supplies for its own load, it is possible that there is no current on the transmission line by choosing certain control. In fact, two subsystem could back up each other, which increases the stability for the whole system. Besides, by detecting the current on the transmission line, one can determine whether the subsystem has problem.

Another direction is to set constraints for battery. For example, battery has a limited capacity, and charging current has a maximum value. Grid-connected DC-microgrid is also an interesting area to study. The DC-microgrid can connect to the power grid with 


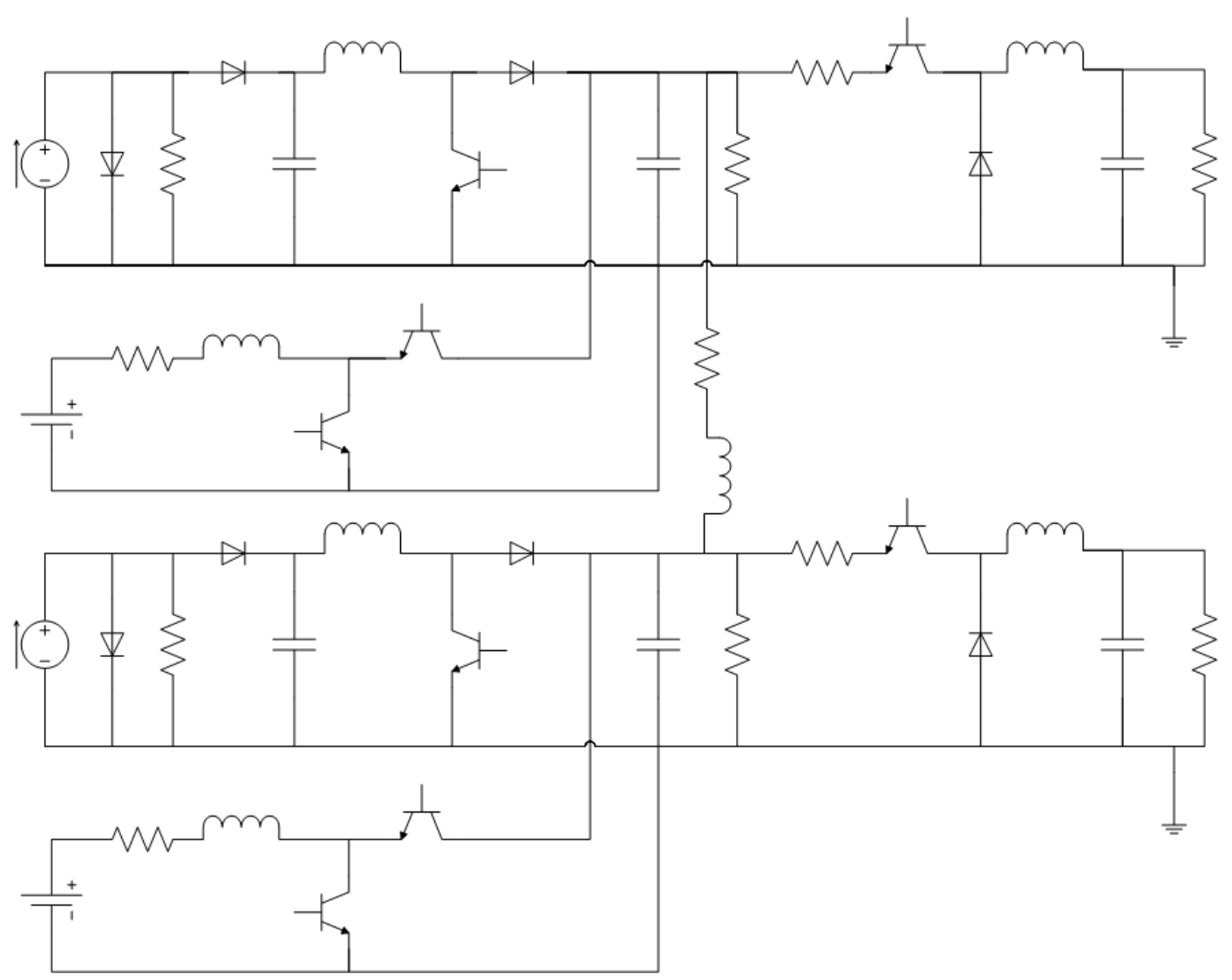

Figure 7.1. The DC-microgrid system with two PVs and batteries

DC/AC converter. At day time, the energy generated from the PV can provide for the grid while the DC-microgrid absorbs power from the power grid at night.

\subsection{More Advanced Control}

Adaptive control can deal with the system with unknown constant parameters. For example, if the values of some resistors are unknown, adaptive control will help. Robust control are designed to deal with the system with uncertain parameters that within some set. For a certain PV, the photogenerated current is within a known range. Therefore, robust control can help with the uncertainty of $I_{p h}$. Adaptive control and robust control have similar function as output regulator theory that they are all used for system 
with unknown disturbances. The output regulator theory can only get local result while adaptive control and robust control may obtain global solution.

Another research area is to study the sensitive of the system. In practice, some of the parameters in the system can only be estimated. Thus, it's important to know whether the designed controller is robust. Robustness can be defined as the ability of a system to resist change without adapting its initial stable configuration. Fortunately, the output regulator theory has a robust version and the passivity-based design is robust too. 


\section{Conclusion}

This work has developed a dynamic model for a DC-microgrid system that consists of a PV, battery, constant resistance load and constant power load. The control objectives are to regulate the bus and load voltage, and to maximize the PV power output.

The stability of the system is analyzed. The DC-microgrid system is a nonlinear nonaffine system which is globally asymptotically stable when using constant control. If using the ideal model of a battery, the system becomes stable in the sense of Lyapunov, but not asymptotically stable. In this case, passivity-based design can help globally asymptotically stabilize the system by arbitrarily small smooth state feedback.

Using both traditional control method and output regulator theory, the controllers are derived for the voltage regulation. In the traditional control method, using the physical meaning of state equations, one can prove that the constant control works. However, constant control may lead to bad dynamic performance. The output regulator theory is useful when dealing with regulation problems. Using output regulator theory, the same equation is derived as the traditional method, which verifies the result. The state feedback control is solved at first, but the state it uses is unmeasurable. Then, the measurement feedback control is developed. The result using output regulator theory is a local 
result due to the use of linear approximation. Finally, a globally controller is derived by passivity-based design.

The controllers are confirmed by simulation results. For both illumination change and load change, controllers using constant control, state feedback control and measurement feedback control are tested. It is shown that constant control will lead to bad dynamic performance which can be solved by state feedback control and measurement feedback control.

The future directions are discussed. Three possible directions are adding disturbance to the system, studying more complexed system and using more advanced control. 


\section{Complete References}

[1] Agarwal, A., Deekshitha, K., Singh, S., \& Fulwani, D. (2015). Sliding mode control of a bidirectional DC/DC converter with constant power load. 2015 IEEE First International Conference on DC Microgrids (ICDCM). doi:10.1109/icdcm.2015.7152056

[2] Ahmadi, R., \& Ferdowsi, M. (2014). Improving the Performance of a Line Regulating Converter in a Converter-Dominated DC Microgrid System. IEEE Transactions on Smart Grid, 5(5), 2553-2563. doi:10.1109/tsg.2014.2319267

[3] Backhaus, S. N., Swift, G. W., Chatzivasileiadis, S., Tschudi, W., Glover, S., Starke, M., ... Hammerstrom, D. (2015). DC Microgrids Scoping Study. Estimate of Technical and Economic Benefits. doi:10.2172/1209276

[4] Becherif, M., Paire, D., \& Miraoui, A. (2007). Energy management of solar panel and battery system with passive control. 2007 International Conference on Clean Electrical Power. doi:10.1109/iccep.2007.384179

[5] Byrnes, C. I., Priscoli, F. D., \& Isidori, A. (1997). Output regulation of uncertain nonlinear systems. Boston: Birkhauser.

[6] Freeman, D. (2010). Introduction to Photovoltaic Systems Maximum Power Point Tracking.

[7] Gautam, A. R., Singh, S., \& Fulwani, D. (2015). DC bus voltage regulation in the presence of constant power load using sliding mode controlled dc-dc Bi-directional converter interfaced storage unit. 2015 IEEE First International Conference on DC Microgrids (ICDCM). doi:10.1109/icdcm.2015.7152050

[8] Gietl, E. B., Gholdston, E. W., Manners, B. A., \& Delventhal, R. A. (2000). The electric power system of the international space station-a platform for power technology development. In Aerospace Conference Proceedings, 2000 IEEE (Vol. 4, pp. 47-54). IEEE.

[9] Kwasinski, A., \& Krein, P. T. (2007). Stabilization of constant power loads in DcDc converters using passivity-based control. INTELEC 07 - 29th International Telecommunications Energy Conference. doi:10.1109/intlec.2007.4448903

[10] Kwasinski, A., \& Onwuchekwa, C. N. (2011). Dynamic Behavior and Stabilization of DC Microgrids With Instantaneous Constant-Power Loads. IEEE Transactions on Power Electronics, 26(3), 822-834. doi:10.1109/tpel.2010.2091285 
[11] Lin, W. (1996). Global asymptotic stabilization of general nonlinear systems with stable free dynamics via passivity and bounded feedback. Automatica, 32(6), 915924.

[12] Radwan, A. A., \& Mohamed, Y. A. (2012). Linear Active Stabilization of ConverterDominated DC Microgrids. IEEE Transactions on Smart Grid, 3(1), 203-216. doi:10.1109/tsg.2011.2162430

[13] Rodriguez, C., \& Amaratunga, G. (n.d.). Dynamic stability of grid-connected photovoltaic systems. IEEE Power Engineering Society General Meeting, 2004. doi:10.1109/pes.2004.1373271

[14] Tan, Y., Kirschen, D., \& Jenkins, N. (2004). A Model of PV Generation Suitable for Stability Analysis. IEEE Transactions on Energy Conversion, 19(4), 748-755. doi:10.1109/tec.2004.827707

[15] Wang, L., \& Lin, Y. (n.d.). Dynamic stability analyses of a photovoltaic array connected to a large utility grid. 2000 IEEE Power Engineering Society Winter Meeting. Conference Proceedings (Cat. No.00CH37077). doi:10.1109/pesw.2000.850010

[16] Wu, T., Kuo, C., Lin, L.,\& Chen, Y. (2016). DC-Bus Voltage Regulation for a DC Distribution System With a Single-Phase Bidirectional Inverter. IEEE Journal of Emerging and Selected Topics in Power Electronics, 4(1), 210-220. doi:10.1109/jestpe.2015.2485300

[17] Zhang, F, Yang, Y., Ji, C., Wei, W., Chen, Y., Meng, C., . . . Zhang, G. (2015). Power management strategy research for DC microgrid with hybrid storage system. 2015 IEEE First International Conference on DC Microgrids (ICDCM). doi:10.1109/icdcm.2015.7152011 